\title{
A Holomorphic Point of View about Geodesic Completeness
}

\author{
Claudio MENEGHINI \\ University of Parma, Department of Mathematics, Strada M.D'Azeglio 85 \\ 43100 Parma, Italy \\ E-mail: clamengh@bluemail.ch
}

Received April 11, 2003; Accepted February 25, 2004

\begin{abstract}
We propose to apply the idea of analytical continuation in the complex domain to the problem of geodesic completeness. We shall analyse rather in detail the cases of analytical warped products of real lines, these ones in parallel with their complex counterparts, and of Clifton-Pohl torus, to show that our definition sheds a bit of new light on the behaviour of 'singularities' of geodesics in space-time. We also show that some geodesics, which 'end' at finite time in the classical sense, can be naturally continued besides their ends. As a matter of fact, complex metrics naturally show a meromorphic behaviour, or a degenerating one, so we shall study also this fact in detail.
\end{abstract}

\section{Foreword}

Within the framework of Riemannian geometry, geodesic and metric completeness are well known to be equivalent: this is Hopf-Rinow's theorem, a consequence of the positivity of Riemannian metrics.

This equivalence does not hold for semi-Riemannian metrics, and there exist even compact lorentzian manifolds which are geodesically incomplete: a well known exemple is Clifton-Pohl torus (see e.g. [ONE], 7.16).

In this paper we propose a definition of geodesic completeness from a complex point of view, that is to say we shall look rather at complexified pseudo-Riemannian manifolds with complex-symmetric metrics.

By a philosophical point of view, our goal is to shed a little bit of light on the behaviour of 'singularities' of geodesics in space-time and show that some geodesics, which seem to 'end' at finite time can be naturally continued besides their ends. This will be done by running along complex trips close to the real line.

Since our approach will use complex-analytical methods and analytical continuation leads in general to poles and zeroes, we shall need the idea of a meromorphic metric on a complex manifold M (see [LEB] for the definition of a holomorphic metric; see also [MAN]; see $[\mathrm{BFV}]$ about the relationship with anti-Kähler geometry; see [CSB] and $[\mathrm{CSC}]$ for physical 
motivations). This will amount to a possibly degenerating symmetric meromorphic section of the twice covariant holomorphic tensor bundle $\mathcal{T}_{0}^{2} \mathrm{M}$.

Of course, it carries no 'signature'. However, by simmetry, it induces a canonical meromorphic Levi-Civita's connexion on M, allowing to define geodesics as the auto-parallel paths. For the sake of completeness this aspects will be dealt with in some details.

It is worth noticing that, if M arises as a 'complexification' of a semi-Riemannian manifold $\mathrm{N}$, it is easily seen that the real geodesics of $\mathrm{N}$ are restrictions to the real axis of the complex ones of $\mathrm{M}$ and vice versa (see [LEB]).

This fact sometimes allows us to 'flank' isolated singularities on the real line, i.e. to 'connect' geodesics which, in the usual sense, are completely unrelated.

The problem of lorentzian geodesic completeness is investigated in $[\mathrm{BEH}],[\mathrm{ONE}],[\mathrm{CR}]$, $[\mathrm{RS}]$.

We suggest a more formal idea of our notion of completeness (see also definition 7) : given a complexification $d: \mathrm{N} \rightarrow \mathrm{M}$ and a real analytic curve $\gamma:[a, b] \rightarrow \mathrm{N}, \gamma$ will be told to be complete provided that $d \circ \gamma$ can be continued to all points in the real line, with at most a discrete set of exceptional values, taking 'real values' (i.e. in $d(\mathrm{~N})$ ).

Finally, we report the main existence-and-uniqueness theorem of ordinary differential equation theory in the complex domain: let $W_{0}$ be a complex $N$-tuple, $z_{0} \in \mathbb{C}$; let $F$ be a $\mathbb{C}^{N}$-valued holomorphic mapping in $\prod_{j=1}^{N} \mathbb{D}\left(W_{0}^{j}, b\right) \times \mathbb{D}\left(z_{0}, a\right),(a, b \in \mathbb{R})$ with $C^{0}$-norm $M$ and $C^{0}$-norm of each $\partial F / \partial w^{j}(j=1 . . N)$ not exceeding $K \in \mathbb{R}$.

Theorem 1. If $r<\min (a, b / M, 1 / K)$, there exists a unique holomorphic mapping $W: \mathbb{D}\left(z_{0}, r\right) \rightarrow \prod_{j=1}^{N} \mathbb{D}\left(W_{0}^{j}, b\right)$ such that $W^{\prime}=F(W(z), z)$ and $W\left(z_{0}\right)=W_{0}$. (see e.g. [HIL], th 2.2.2, [INC] p.281-284)

NB: in the following we shall abbreviate 'holomorphic function element', resp. 'holomorphic function germ' by HFE resp. HFG.

\section{Analytical continuation}

In the following, $\mathcal{U}$ will be a region in the complex plane and $\mathrm{M}$ a complex manifold: the idea of the analytical continuation of a holomorphic mapping element (or of a germ) $f: \mathcal{U} \rightarrow \mathrm{M}$ is well known see e.g. [CAS], chap. 5, rather than [FO], 1.7-1.8) and amounts to a quadruple $Q_{\mathrm{M}}=(S, \pi, j, F)$, where $S$ is a connected Riemann surface over a region of $\mathbb{C}, \pi: S \rightarrow \mathbb{C}$ is a nonconstant holomorphic mapping such that $U \subset \pi(S), j: U \rightarrow S$ is a holomorphic immersion such that $\pi \circ j=\left.i d\right|_{U}$ and $F: S \rightarrow$ M is a holomorphic mapping such that $F \circ j=f$. Each finite branch point is kept into account by the fact of lying 'under' some critical point of $\pi$ : for example, the Riemann surface of $\left.\sqrt{z}\right|_{D(1,1 / 2)}$, with $\sqrt{1}=1$, is $\left(\mathbb{C}, \zeta \mapsto \zeta^{2},\left.\sqrt{z}\right|_{D(1,1 / 2)}, \zeta \mapsto \zeta\right)$ : the double branch point $z=0$ of the analytical continuation lies under the critical point $\zeta=0$, branching being taken into account by the squaring function.

A morphism between two analytical continuations $(S, \pi, j, F)$ and $(T, \varrho, \ell, G)$ of the same element $(U, f)$ is a holomorphic mapping $h: T \rightarrow S$ such that $h \circ \ell=j$. Note that a morphism between two analytical continuations is a nonconstant (in particular open) mapping, uniquely determined on $j(U)$, hence everywhere on $S$, by $\ell \circ j^{-1}$. Moreover, $\varrho \circ h=\pi$ and $G \circ h=F$ on $j(U)$, hence everywhere on $S$. 
The only existing morphism between an analytical continuation and itself is the identity mapping; the composition of two morphisms is a morphism; if a morphism admits a holomorphic inverse mapping, this is a morphism too: in such a case we talk about isomorphisms of analytical continuations.

Definition 1. An analytical continuation $S$ of the element $(U, f)$ is maximal if for every regular analytical continuation $\widehat{S}$ of $(U, f)$ there exists a morphism $h: S \rightarrow \widehat{S}$.

Two maximal continuations of the same element must be isomorphic, since they admit morphisms one into each other; thus the maximal regular analytical continuation of an element is unique up to isomorphisms.

The following is a well known result (we refer to [CAS], th.5.6.4, pages 262-266).

Theorem 2. Every element $(U, f)$ (hence every germ) of holomorphic function admits a maximal analytical continuation, called the Riemann surface, of $(\mathcal{U}, f)$.

Definition 2. A logarithmic singularity $q$ of $Q_{\mathrm{M}}=(S, \pi, j, F)$ (in the following: $L$ singularity) is a decreasing sequence of open sets $\left\{V_{k}\right\}_{k \geq K}$ of $S$ such that:

- (LS1) for every $k \geq K, V_{k}$ is a connected component of $\pi^{-1}\left(D\left(z_{0}, \frac{1}{k}\right) \backslash\left\{z_{0}\right\}\right)$ and $\left.\pi\right|_{V_{k}}$ is a topological covering of $\left(D\left(z_{0}, \frac{1}{k}\right)\right.$;

- (LS2) $\bigcap_{k \geq K} \overline{V_{k}}=\emptyset$;

- (LS3) for every $k \geq K$ and every (real) nonconstant closed path $\gamma:[0,1] \rightarrow$ $D\left(z_{0}, 1 / k\right) \backslash\left\{z_{0}\right\}$, with nonzero winding number around $z_{0}$, every lifted path $\tilde{\gamma}:[0,1] \rightarrow$ $\pi^{-1}\left(D\left(z_{0}, 1 / k\right) \backslash\left\{z_{0}\right\}\right)$ is such that $\tilde{\gamma}(0) \neq \tilde{\gamma}(1)$;

- (LS4) there exists $m \in \mathrm{M}$ such that $\bigcap_{k \geq K} \overline{F\left(V_{k}\right)}=m$.

Consider now the set $B$ of the L-singularities of $Q_{\mathrm{M}}$ : let $S^{\sharp}:=S \bigcup B$ as a set and introduce a topology on $S^{\sharp}$ : open sets are the open sets in $S$ and a fundamental neighbourhood system of the L-singularity $q=\left\{V_{k}\right\}_{k \geq K} \in B$ is yielded by the sets $V_{k}^{\sharp}=V_{k} \bigcup\{q\}$. It is easily seen that $S^{\sharp}$ admits no complex structure at $q=\left\{V_{k}\right\}_{k \geq K}$. Indeed, were there one, we could find charts $(\mathcal{W}, \phi)$ around $q$ and $(\mathcal{V}, \psi)$ around $z_{0}$ such that $\psi \circ \pi \circ \phi^{-1}(\zeta)=\zeta^{N}$ for some integer $N>0$. This fact would imply $\left.\pi\right|_{\mathcal{W} \backslash\{q\}}$ to be a n-sheeted covering of $\mathcal{V} \backslash\left\{z_{0}\right\}$; it is easily seen tha this fact would contradict (LS2) in definition 2 .

Lemma 1. (A): $\pi$ admits a unique continuous extension $\pi^{\sharp}$ to $S^{\sharp}$; (B): for every logarithmic singularity $r$ of $Q_{\mathrm{M}}, F$ admits a unique continuous extension $F^{\sharp}$ to $r$.

Proof. (A): let $b \in B$ and $\left\{V_{k}\right\}$ be the sequence spotting $b$ : define $\pi^{\sharp}(q)=\pi(q)$ if $q \in V_{k}$ and $\pi^{\sharp}(b)=z_{0}$, where $z_{0}$ is the common centre of the discs onto which the $V_{k}^{\prime} s$ are projected. Now $\pi^{\sharp}$ is continuous at all points in $V_{k}$; moreover, for every neighbourhood $G$ of $z_{0}, \pi^{\sharp-1}(G) \supset \pi^{\sharp-1}\left(z_{0}\right) \bigcup \pi^{-1}\left(G \backslash\left\{z_{0}\right\}\right)$, hence, if we set $H=\{b\} \bigcup \pi^{-1}\left(G \backslash\left\{z_{0}\right\}\right)$, we have that $H$ is a neighbourhood of $b$ in $S^{\sharp}$ such that $\pi^{\sharp}(H) \subset G$, proving continuity at $b$. Arguing by density, we conclude that this extension is unique; the proof of (B) is analogous.

Definition 3. A quadruple $Q_{\mathrm{M}}^{\natural}=\left(S^{\natural}, \pi^{\natural}, j^{\natural}, F^{\natural}\right)$, is an analytical continuation with $L$ singularities of the function element $(U, f)$ if there exists an analytical continuation $Q_{\mathrm{M}}$ of $(U, f)$ such that $S^{\natural} \backslash S$ consists of L-singularities of $F, \pi^{\natural}$ is the unique continuous extension of $\pi$ to $S^{\natural}, j^{\natural}=i d_{S \rightarrow S^{\natural}} \circ j$ and $F$ admits a unique continuous extension $F^{\natural}$ to 
$S^{\natural}$. $Q_{\mathrm{M}}^{\natural}$ is: maximal provided that so is $Q_{\mathrm{M}}$ and $Q_{\mathrm{M}}^{\natural} \backslash Q_{\mathrm{M}}$ contains all L-singularities of $Q_{\mathrm{M}}$.

Lemma 2. (1): let $\boldsymbol{f}$ and $\boldsymbol{g}$ be two complex-valued holomorphic germs, each one inverse of the other; let $(R, \pi, j, F)$ and $(S, \rho, \ell, G)$ be their respective Riemann surfaces: then $F(R)=\rho(S)$;

(2): let $\boldsymbol{f}, \boldsymbol{g}, \boldsymbol{h}$ be three HFG's such that $\boldsymbol{f} \circ \boldsymbol{g}=\boldsymbol{h}$. Let $(R, \pi, j, F)$ be the Riemann surface of $\boldsymbol{f},(S, \rho, \ell, G)$ the one of $\boldsymbol{g}$ and $(T, \sigma, m, H)$ the Riemann surface with L-singularities of $\boldsymbol{h}$ : then $F(R) \backslash(\mathbb{C} \backslash(\sigma(T))) \subset \rho(S)$.

Proof. (1) a): $F(R) \subset \rho(S)$ : let $\xi \in R$ and $F(\xi)=\eta$; there exist: an open neighbourhood $\mathcal{U}_{1}$ of $\xi$; open subsets $\mathcal{U}_{2} \subset \pi\left(\mathcal{U}_{1}\right)$ and $\mathcal{V}_{2} \subset F\left(\mathcal{U}_{1}\right)$ and a biholomorphic function $g_{2}: \mathcal{V}_{2} \rightarrow \mathcal{U}_{2}$, with inverse function $f_{2}: \mathcal{U}_{2} \rightarrow \mathcal{V}_{2}$ such that: $\left(\mathcal{U}_{2}, f_{2}\right)$ and $(\mathcal{U}, f)$ are connectible and so are $\left(\mathcal{V}_{2}, g_{2}\right)$ and $(\mathcal{V}, g)$. By construction there hence exist two holomorphic immersions $\widetilde{\mathrm{\jmath}}: \mathcal{U}_{2} \rightarrow R$ and $\tilde{\ell}: \mathcal{V}_{2} \rightarrow S$ such that $\pi \circ \widetilde{\jmath}=\boldsymbol{i d}$ and $\rho \circ \tilde{\ell}=\boldsymbol{i d}$. Let $\mathcal{V}_{1}=F(U)_{1}$ and $\Sigma=\left\{(x, y) \in \mathcal{U}_{1} \times \mathcal{V}_{2}: F(x)=y\right\}$; moreover let $J: \mathcal{V}_{2} \rightarrow \Sigma$ be defined by setting $J(v)=\left(\tilde{\mathrm{J}} \circ g_{2}(v), v\right)$. Then $\left(\Sigma, p r_{2}, J, \pi \circ p r_{1}\right)$ is an analytical continuation of $\left(\mathcal{V}_{2}, g_{2}\right)$; indeed $\pi \circ p r_{1} \circ J=\pi \circ \tilde{\mathrm{j}} \circ g_{2}=g_{2}$. But $\left(\mathcal{V}_{\in}, g_{2}\right)$ is connectible with $(\mathcal{V}, g)$, hence $\left(\Sigma, p r_{2}, J, \pi \circ p r_{1}\right)$ is an analytical continuation of $(\mathcal{V}, g)$. Eventually, there exists a holomorphic function $h: \Sigma \rightarrow S$ such that $\rho \circ h=p r_{2}$ : hence $\eta=p r_{2}(\xi, \eta)=\rho \circ h(\xi, \eta) \in \rho(S)$.

b) : $\rho(S) \subset F(R)$ : let $s \in S$ : there is a neighbourhood $V$ of $s$ in $S$ such that $V \backslash\{s\}$ consists entirely of regular points both of $\rho$ and $G$, not excluding that $s$ itself be regular for $\rho$ or $G$ or both. This fact means that for each $s^{\prime} \in V \backslash\{s\}$ there exists a HFE $\left(\rho\left(s^{\prime}\right), \mathcal{V}^{\prime}, \widetilde{g}_{s^{\prime}}\right)$ connectible with $(\mathcal{V}, g)$ and, besides, a holomorphic immersion $\widetilde{\ell}: \mathcal{V}^{\prime} \rightarrow V$. By a) : already proved, $G(s) \in \pi(R)$, hence there exist $p \in R$ such that $\pi(p)=G(s)$ and a neighbourhood $W$ of $p$ in $R$ such that $\pi^{-1}\left(\widetilde{g}\left(\mathcal{V}^{\prime}\right)\right) \bigcap W \neq \emptyset$. Set $W^{\prime}=\pi^{-1}\left(\widetilde{g}\left(\mathcal{V}^{\prime}\right)\right) \bigcap W$ : we may suppose, without loss of generality, that $\pi$ is invertible on $W^{\prime}$ : hence there exists a (open) holomorphic immersion $\widetilde{\jmath}: \widetilde{g}\left(\mathcal{V}^{\prime}\right) \rightarrow W$. Therefore, for each $\zeta \in \widetilde{\jmath}\left(\widetilde{g}\left(\mathcal{V}^{\prime}\right)\right)$, there exists $\eta \in \widetilde{\ell}\left(\mathcal{V}^{\prime}\right)$ such that $F(\zeta)=F(\widetilde{\mathrm{J}} \widetilde{g} \circ \rho(\eta))$. Now, by definition of analytical continuation there holds $F \circ \widetilde{\mathrm{j}} \circ \widetilde{g}=\boldsymbol{i d}$, hence we have $F(\zeta)=\rho(\eta)$. Consider now the holomorphic function $\Xi: W \times V \rightarrow \mathbb{C}$ defined by setting $\Xi(w, v)=F(w)-\rho(v)$ : we have $\Xi \equiv 0$ on the open set $\widetilde{\mathrm{J}}\left(\widetilde{g}\left(\mathcal{V}^{\prime}\right)\right) \times \widetilde{\ell}\left(\mathcal{V}^{\prime}\right)$, thus $\Xi \equiv 0$ on $W \times V$ : this in turn implies $F(p)=\rho(s)$. Therefore we have proved that for each $s \in S$ there exists $p \in R$ such that $F(p)=\rho(s)$ : this eventually implies that $\rho(S) \subset F(R)$.

(2): let $\xi \in R$ such that $\eta=F(\xi) \notin \mathbb{C} \backslash(\sigma(T))$ : there exist: an open neighbourhood $\mathcal{U}_{1}$ of $\xi$, open subsets $\mathcal{U}_{2} \subset \pi\left(\mathcal{U}_{1}\right), \mathcal{V}_{2} \subset F\left(\mathcal{U}_{1}\right)$ and $\mathcal{W}_{2} \subset \sigma(T)$ and biholomorphic functions $f_{2}: \mathcal{U}_{2} \rightarrow \mathcal{W}_{2}, g_{2}: \mathcal{V}_{2} \rightarrow \mathcal{U}_{2}$ and $h_{2}: \mathcal{V}_{2} \rightarrow \mathcal{W}_{2}$ such that: $\left(\mathcal{U}_{2}, f_{2}\right)$ and $\boldsymbol{f}$ are connectible, $\left(\mathcal{V}_{2}, g_{2}\right)$ and $\boldsymbol{g}$ are connectible, $\left(\mathcal{V}_{2}, h_{2}\right)$ and $\boldsymbol{h}$ are connectible, and $f_{2} \circ g_{2}=h_{2}$.

By construction there hence exist three holomorphic immersions $\widetilde{\mathrm{j}}: \mathcal{U}_{2} \rightarrow R, \tilde{\ell}: \mathcal{V}_{2} \rightarrow S$, $\widetilde{m}: \mathcal{W}_{2} \rightarrow T$ such that $\pi \circ \widetilde{\mathrm{j}}=\boldsymbol{i d}, \rho \circ \widetilde{\ell}=\boldsymbol{i d}$ and $\sigma \circ \widetilde{m}=\boldsymbol{i d}$.

Let $\mathcal{V}_{1}=F\left(U_{1}\right), \mathcal{W}_{1}$ be the connected component of $\sigma^{-1}\left(F\left(\mathcal{U}_{1}\right)\right)$ in $T$ and $\Sigma=$ $\left\{(x, y) \in \mathcal{U}_{1} \times \mathcal{W}_{1}: F(x)=H(y)\right\}$; moreover let $J: \mathcal{V}_{2} \rightarrow \Sigma$ be defined by setting $J(v)=\left(\widetilde{\mathrm{J}} \circ g_{2}(v), \widetilde{m}(v)\right)$.

Then $\left(\Sigma, p r_{2}, J, \pi \circ p r_{1}\right)$ is an analytical continuation, with logarithmic singularities of $\left(\mathcal{V}_{2}, g_{2}\right)$; indeed $\pi \circ p r_{1} \circ J=\pi \circ \widetilde{\mathrm{j}} \circ g_{2}=g_{2}$; but $\left(\mathcal{V}_{\in}, g_{2}\right)$ is connectible with $(\mathcal{V}, g)$, hence $\left(\Sigma, p r_{2}, J, \pi \circ p r_{1}\right)$ is an analytical continuation of $(\mathcal{V}, g)$. 
Thus there exists a continuous function $h: \Sigma \rightarrow S$, holomorphic on the interior of $\Sigma$, such that $\rho \circ h=p r_{2}$ : hence $\eta=p r_{2}(\xi, \eta)=\rho \circ h(\xi, \eta) \in \rho(S)$.

\section{Paths}

Let's start with a slight reformulation of the notion of path: to achieve this goal, we adopt the point of view according to which a 'path' or even a 'curve' are analytical continuations of some initial germs, generally yielded by local solutions of systems of differential equations.

We shall also deal with the velocity field of a path: to define it we shall need to single out a vector field on its domain of definition, which will have to be related with the natural derivation field $d / d z$ on $\mathbb{C}$.

Let $\mathrm{M}$ be a connected complex manifold: in the continuation, abusing language but following Wells (see e.g. [WEL] or [GRO]), we shall name $T \mathrm{M}$ (resp.T* $T^{*}$ ) its holomorphic tangent (resp. cotangent) bundle and, more generally, $\mathcal{T}_{r}^{s} \mathrm{M}$ its holomorphic r-covariant and s-contravariant tensor bundle; as usual, $\Pi: \mathcal{T}_{r}^{s} \mathrm{M} \rightarrow \mathrm{M}$ will denote the natural projection.

A closed hypersurface $\mathcal{F}$ in $M$ is a closed subset such that there exists a maximal atlas $\left\{U_{n}\right\}$ for $\mathrm{M}$ and, for each $n$, a holomorphic function $\Psi_{n}$, not vanishing everywhere, such that $U_{n} \bigcap \mathcal{F}=\left\{X \in U_{n}: \Psi_{n}(X)=0\right\}$.

The following definition is adapted from [ONE], definition 2.4 and lemma 2.5:

Definition 4. let $\mathcal{E}$ be a closed hypersurface in $\mathrm{M}, \mathrm{N}$ another connected complex manifold and $F \in \mathcal{O}(\mathrm{M}, \mathrm{N})$ : an $\mathcal{E}$-meromorphic section of $\mathcal{T}_{r}^{s} \mathrm{~N}$ over $F$ is a holomorphic section $\Lambda$ of $\mathcal{T}_{r}^{s} \mathrm{~N}$ over $\left.F\right|_{\mathrm{M} \backslash \mathcal{E}}$ such that $\pi \circ \Lambda$ admits analytical continuation up to the whole $\mathrm{M}$ and for every $p \in \mathcal{E}$ and every coordinate system $\left(\mathcal{U},\left(z^{1} \ldots z^{n}\right)\right)$ around $F(p)$, there exists a neighbourhood $U$ of $p$ and $r \cdot s$ pairs of $\mathbb{C}$-valued holomorphic functions $\phi_{i_{1} \ldots i_{r}}, \psi_{l_{1} \ldots l_{s}}$, with $\psi_{l_{1} \ldots l_{s}} \neq 0$ on $U \backslash \mathcal{E}$, such that

$$
\Lambda\left(d z^{l_{1}} \ldots d z^{l_{s}}, \frac{\partial}{\partial z^{i_{1}}} \ldots \frac{\partial}{\partial z^{i_{r}}}\right)=\frac{\phi_{i_{1} \ldots i_{r}}}{\psi_{l_{1} \ldots l_{s}}} .
$$

Definition 5. A path in $\mathrm{M}$ is a quadruple $Q_{\mathrm{M}}=(S, \pi, j, F)$, where $S$ is a connected Riemann surface, $\pi: S \rightarrow \mathbb{C}$ is a branched covering of $S$ over $\pi(S), F: S \rightarrow \mathrm{M}$ is a holomorphic mapping, $U \subset \mathbb{C}$ is an open set wich admits a holomorphic (hence open) immersion $j: U \rightarrow S \backslash \Sigma$ such that $\pi \circ j=\left.i d\right|_{U}$.

We are now turning to define the velocity field of a path $Q_{\mathrm{M}}$ : it will be defined as a suitable meromorphic section over $F$ of the holomorphic tangent bundle TM. To achieve this purpose, we need to lift the vector field $d / d z$ on $\mathbb{C}$ with respect to $\pi$.

Of course, in general, contravariant tensor fields couldn't be lifted; notwithstanding, we may get through this obstruction by keeping into account that $\mathbb{C}$ and $S$ are one-dimensional manifolds and allowing the lifted vector field to be meromorphic: these matters are fathomed in next statements: recall that $P$ is the set of branch points of $\pi$.

Lemma 3. There exists a unique $P$-meromorphic vector field $\widetilde{d / d z}$ on $S$ such that, for every $r \in S \backslash P,\left.\pi_{*}\right|_{r}\left(\left.\widetilde{d / d z}\right|_{r}\right)=\left.(d / d z)\right|_{\pi(r)}$. 
Proof. Consider $\omega=\pi^{*} d z$ and $\Lambda=\pi^{*}(d z \odot d z)$ on $S$ : the latter establishes an isomorphism between the holomorphic cotangent and tangent bundles of $S \backslash P$. Call $V$ the holomorphic vector field corresponding to $\omega$ in the above isomorphism: we claim that $V=\widehat{d / d z}$ on $S \backslash P$. To show this fact, we explicitely compute the components of $V$ with respect to a maximal atlas $\mathcal{B}=\left\{\left(U_{\nu}, \zeta_{\nu}\right)\right\}$ for $S \backslash P$ : let $\omega_{(\nu) 1}=\omega\left(\partial / \partial \zeta_{(\nu)}\right)$, $g_{(\nu) 11}=\Lambda\left(\partial / \partial \zeta_{(\nu)}, \partial / \partial \zeta_{(\nu)}\right)$; then, set $V_{(\nu)}^{1}=\omega_{(\nu) 1} / g_{(\nu) 11}$ the collection $\left\{\left(\mathcal{U}_{\nu}, V_{(\nu)}^{1}\right)\right\}$ of open sets and holomorphic functions is such that, on overlapping local charts $\left(U_{a}, \zeta_{a}\right)$ and $\left(U_{b}, \zeta_{b}\right)$, we have

$$
V_{(a)}^{1}=\frac{\omega_{(a) 1}}{g_{(a) 11}}=\frac{\omega_{(b) 1}\left(d \zeta_{(b)} / d \zeta_{(a)}\right)}{g_{(b) 11}\left(d \zeta_{(b)} / d \zeta_{(a)}\right)^{2}}=V_{(b)}^{1} \frac{d \zeta_{(a)}}{d \zeta_{(b)}},
$$

that is to say that collection defines a holomorphic vector field. Now for every $r \in S \backslash P$,

$$
\left.d z\right|_{\pi(r)}\left(\left.\left.\pi_{*}\right|_{r} \widetilde{d / d z}\right|_{r}\right)=\left.\pi^{*} d z\right|_{r}\left(\left.\widetilde{d / d z}\right|_{r}\right)=\frac{\left.\pi^{*} d z\right|_{r}\left(\partial /\left.\partial \zeta\right|_{r}\right)}{\left.d z\right|_{\pi(r)}\left(\pi_{*} \partial /\left.\partial \zeta\right|_{r}\right)}=1
$$

hence $\left.\pi_{*}\right|_{r}\left(\left.\widetilde{d / d z}\right|_{r}\right)=\left.(d / d z)\right|_{\pi(r)}$, proving the asserted.

Let's prove that $\widetilde{d / d z}$ may be extended to a meromorphic vector field on $S$ : if $p \in P$ then we can find local charts $(U, \psi)$ around $p,(V, \phi)$ around $\pi(p)$, and an integer $N>0$ such that $\phi \circ \pi \circ \psi^{-1}(u)=u^{N}$. Now we have

$$
\left.\left.\left(\psi^{-1 *} \pi^{*} \phi^{*}(d w) \frac{d}{d u}\right)(u)=\left.d w\left(\phi_{*} \pi_{*} \psi_{*}^{-1} \frac{d}{d u}\right)\right|_{u}\right)=d w\left(\left(\phi \pi \psi^{-1}\right)^{\prime} \frac{d}{d w}\right)\right)=N u^{N-1} ;
$$

but $\phi$ and $\psi$ are charts, hence $\pi^{*} d z$ itself is vanishing of order $N-1$ at $p$; as already proved, $\left.\pi_{*}\right|_{r}\left(\left.\widetilde{d / d z}\right|_{r}\right)=\left.(d / d z)\right|_{\pi(r)}$ on $U \backslash\{p\}$ and, consequently, $\left(\pi^{*} d z\right)(\widetilde{d / d z})=d z(d / d z)=1$ on $U \backslash\{p\}$, hence on $U$. Now, in local coordinates, $\left(\pi^{*} d z\right)=\alpha d \phi$ and $\widetilde{d / d z}=y \partial / \partial \phi$, where $\alpha$ is a suitable holomorphic function on $U$, vanishing of order $N-1$ at $p$ and $y$ is a holomorphic function on $U \backslash\{p\}$. By the above argument, $y \alpha=1$, hence $y$ has a pole of order $N-1$ at $p$ : a similar argument holds for each isolated point in $P$, proving the meromorphic behaviour of $\widetilde{d / d z}$.

Definition 6. A finite-velocity point of a path $Q_{\mathrm{M}}=(S, \pi, j, F, \mathrm{M})$ is a point $r \in S$ such that $\widetilde{d / d z}$ is holomorphic at $r$.

We are ready to define the velocity field : let at first be $r$ a finite-velocity point of $Q_{\mathrm{M}}$; since $\widetilde{d / d z}$ is holomorphic at $r$, we could define the holomorphic velocity at $r$ as $\left.V_{r}=\left.F_{*}(\widetilde{(d / d z})\right|_{r}\right):$ now define the mapping $V\left(Q_{\mathrm{M}}\right): S \backslash P \rightarrow T \mathrm{M}$ by setting $r \mapsto$ $\left(F, F_{*}(\widetilde{d / d z}(r))\right)$.

Lemma 4. The mapping $V\left(Q_{\mathrm{M}}\right)$ can be extended to a P-meromorphic section of TM over $F$.

Proof. Trivially $\left.\Pi \circ V\right|_{R \backslash P}=\left.F\right|_{R \backslash P}$. Let's show the meromorphic behaviour of $V$ : if $p \in P$ there is a neighbourhood $U$ of $p$ such that, for every local chart $\zeta: U \rightarrow \mathbb{C}_{w}$ there 
exist holomorphic functions $f, g \in \mathcal{H}(\zeta(U))$ such that

$$
\left.\frac{\widetilde{d}}{d z}\right|_{\zeta_{-1}(U)}=\zeta_{*}^{-1}\left(\left.\frac{f}{g}(w) \frac{d}{d w}\right|_{w}\right) ;
$$

moreover, for every local chart $\Psi=\left(u^{1} \ldots u^{m}, d u^{1} \ldots d u^{m}\right)$ in $T \mathrm{M}$ we obtain

$$
\begin{aligned}
& \Psi \circ V \circ \zeta^{-1}(w)= \\
= & \Psi \circ\left(F \circ \zeta^{-1}(w),\left.F_{*}\right|_{\zeta^{-1}(w)}\left(\left.\frac{\widetilde{d}}{d z}\right|_{\zeta^{-1}(w)}\right)\right) \\
= & \Psi \circ\left(F \circ \zeta^{-1}(w),\left.F_{*}\right|_{\zeta^{-1}(w)} \zeta_{*}^{-1}\left(\frac{f}{g}(w) \frac{d}{d w} \mid w\right)\right) \\
= & \Psi \circ\left(F \circ \zeta^{-1}(w), \frac{f}{g}(w) \frac{d}{d w}\left(F \circ \zeta^{-1}\right)(w)\right) \\
= & \left(u^{1} \circ F \zeta^{-1}(w) \ldots u^{m} \circ F \zeta^{-1}(w),\right. \\
& \left.\left.\frac{f}{g}(w) \frac{d}{d w}\left(u^{1} \circ F \circ \zeta^{-1}\right)(w) \ldots \frac{f}{g}(w) \frac{d}{d w}\left(u^{m} \circ F \circ \zeta^{-1}\right)(w)\right)\right)
\end{aligned}
$$

According to lemma 4, the velocity field of a path $Q_{\mathrm{M}}=(S, \pi, j, F, \mathrm{M})$ will be just the meromorphic mapping $V\left(Q_{\mathrm{M}}\right)$.

\subsection{Definition of completeness}

Definition 7. A M-valued path $(S, \pi, j, F)$ is complex-complete provided that $\mathbb{C} \backslash \pi(S)$ is a finite set in the complex plane; a real-analytic curve $\gamma$ in a real-analytic manifold $\mathrm{N}$ admitting a complexification $d: \mathrm{N} \rightarrow \mathrm{M}$ is real-complete (or, briefly, complete) provided that the Riemann surface $(S, \pi, j, G)$ of $d \circ \gamma$ is such that $\mathbb{R} \backslash \pi\left(G^{-1}(d(\mathrm{~N}))\right)$ is a finite set.

\section{Complex-Riemannian metric structures}

The intuitive geometry of the real euclidean space $\mathbb{R}^{3}$ can be easily brought back to its natural inner product, which allows basic geometrical operations, like measuring the length of a tangent vector, or angles between tangent vectors: Riemannian real geometry generalizes all this to 'curved' spaces, which is based on the concept of positive definite bilinear forms: weakening definiteness to nondegeneracy leads us in the realm of Lorentz geometry, originating from the problems posed by Einstein's general relativity theory.

A little bit less intuitive is the idea of starting from the basic geometry of $\mathbb{C}^{3}$ (meant as a 'complexification' of the usual real euclidean space) to get formal extension of the geometric properties of real 'curved' manifolds. Introducing this complex environment could allow us to hope to get able to handle some types of metrical singularities which naturally arise in dealing with real manifolds with indefinite metrics.

It is immediately seen that the nondegeneracy hypothesis itself should be dropped, as the following considerations show (see $[\mathrm{DNF}]$ p.186 ff): consider the space $\mathcal{F}$ of antisymmetric covariant tensors of rank two in Minkowski's space $\mathbb{R}_{1,3}$ : electromagnetic fields are such 
ones. Let $F \in \mathcal{F}$ : we can write $F=\sum_{i<j} F_{i j} d x^{i} \wedge d x^{j}$ where $x^{0} \ldots x^{3}$ are the natural coordinate functions on $\mathbb{R}_{1,3}$. At each point, the space $\mathcal{F}_{p}$ of all tensors in $\mathcal{F}$ evaluated at $p$ is a six-dimensional real vector space; moreover, the adjoint operator $*$ with respect to Minkowski's metric is such that $* *=-1$ : all these facts imply that $\mathcal{F}_{p}$ could be thought of as a complex three dimensional vector space $\mathcal{G}_{p}$ by setting $(a+\boldsymbol{i} b) F=a F+b * F$. Now * is $S O(1,3)$-invariant, hence $S O(1,3)$ is a group of (complex) linear transformations of $\mathcal{G}_{p}$, preserving the quadratic form $\langle F, F\rangle=-*(F \wedge(* F)+\boldsymbol{i} F \wedge F)$ : this means that this 'norm' is invariant by Lorentz transformations, hence it is of relevant physical interest. If we introduce the following coordinate functions on $\mathcal{G}_{p}: z^{1}=F_{01}-i F_{23}, z^{2}=F_{02}+i F_{13}$ and $z^{1}=F_{03}-i F_{12}$, we have that $\langle F, F\rangle=\left(z^{1}\right)^{2}+\left(z^{2}\right)^{2}+\left(z^{3}\right)^{2}$, hence there naturally arises the so called complex-Euclidean metric on $\mathbb{C}^{3}$ : on one hand, by changing coordinates we are brought to a generic symmetric bilinear form on $\mathbb{C}^{3}$; on the other, there arise 'poles' if we attempt to extend the above construction e.g. to $\left(\mathbb{P}^{1}\right)^{3}$. Now the idea of generalizing to the curved framework is quite natural: let $\mathrm{M}$ be a complex manifold, $\mathcal{D}$ and $\mathcal{E}$ closed hypersurfaces in $\mathrm{M}$.

Definition 8. A holomorphic (resp. $\mathcal{E}$-meromorphic) metric on M is a holomorphic (resp.Emeromorphic) section $\Lambda: \mathrm{M} \rightarrow \mathcal{T}_{0}^{2} \mathrm{M}$ which is symmetric, that is to say, for every $m \in \mathrm{M}$ and every pair of holomorphic tangent vectors $V_{m}$ and $W_{m}$ at $m$, there holds $\Lambda(m)\left(V_{m}, W_{m}\right)=$ $\Lambda(m)\left(W_{m}, V_{m}\right)$;. The rank of $\Lambda$ at $p \in \mathrm{M}$ is the rank of the bilinear form $\Lambda(p) ; \Lambda$ is nondegenerate at $p$ if $r k(\Lambda(p))=\operatorname{dim}(\mathrm{M})$, degenerate otherwise; if $\mathcal{D}$ is a hypersurface in $\mathrm{M}$ and $\Lambda$ is degenerate only on $\mathcal{D}$, we shall say that $\Lambda$ is $\mathcal{D}$-degenerate. We say that $p$ is a metrically ordinary point in $\mathrm{M}$ if $\Lambda$ is holomorphic and nondegenerate at $p$.

In the following we shall consider only metric which degenerate only on closed hypersurfaces.

Definition 9. A holomorphic Riemannian manifold is a complex manifold endowed with a holomorphic metric; a nondegenerate holomorphic Riemannian manifold is a complex manifold endowed with a nondegenerate holomorphic metric ; a meromorphic Riemannian manifold is a complex manifold endowed with a meromorphic metric.

Thus, strictly speaking, all the above objects are pairs consisting in complex manifolds and metrics, but we shall often understand metrics and denote them by the only underlying complex manifolds.

\subsection{The meromorphic Levi-Civita connexion}

We begin this section by introducing the holomorphic Levi-Civita connexion induced on a holomorphic nondegenerate Riemannian manifold by its metric structure: this is done in a quite similar way to that pursued in (real) differential geometry, apart from a slight difference, which naturally arises: the action of the Levi-Civita connexion is defined at first on 'local' vector fields, producing local ones as well, then it is globalized as a collection of local operators.

Let now $(\mathrm{M}, \Lambda)$ be a nondegenerate Riemannian holomorphic manifold, $\mathcal{A}$ a maximal atlas for $\mathrm{M}, \mathcal{U} \in \mathcal{A}$ a domain of a local chart. Let also $\mathcal{X}(\mathcal{U})$ be the Lie algebra of holomorphic vector fields on $\mathcal{U}$ and $\mathcal{O}(\mathcal{U})$ the ring of holomorphic functions on $\mathcal{U}$. 
Definition 10. A connexion on $\mathcal{U}$ is a mapping $D: \mathcal{X}(\mathcal{U}) \times \mathcal{X}(\mathcal{U}) \rightarrow \mathcal{X}(\mathcal{U})$ such that: (D1) $D_{V} W$ is $\mathcal{H}(\mathcal{U})$-linear in $V$; (D2) $D_{V} W$ is $\mathbb{C}$-linear in $W$ and (D3) $D_{V}(f W)=$ $(V f) W+f D_{V} W$ for every $f \in \mathcal{H}(\mathcal{U})$.

$D_{V} W$ is called the covariant derivative of $W$ with respect to $V$ in the connexion $D$. By axiom (D1), $D_{V} W$ has tensor character in $V$, while axiom (D3) tells us that it is not a tensor in $W$.

Our next step is to show that there is a unique connexion characterized by two further properties, (D4) and (D5) below, namely being anti-Leibnitz like with respect to the Lie bracket operation and Leibnitz like with respect to the metric. In the following we use the alternative notation $\langle V, W\rangle$ instead of $\Lambda(V, W)$.

Lemma 5. Let $\mathcal{U}$ be an open set belonging to a maximal atlas $\mathcal{A}$ for the nondegenerate holomorphic Riemannian manifold M. If $V \in \mathcal{X}(\mathcal{U})$, let $V^{*}$ be the holomorphic one-form on $\mathcal{U}$ such that $V^{*}(X)=\langle V, X\rangle$ for every $X \in \mathcal{X}(\mathcal{U})$ : then the mapping $V \mapsto V^{*}$ is a $\mathcal{O}$-linear isomorphism from $\mathcal{X}(\mathcal{U})$ to $\mathcal{X}^{*}(\mathcal{U})$.

Proof. Since $V^{*}$ is $\mathcal{O}$-linear, it is in fact a one-form, and $V \mapsto V^{*}$ is $\mathcal{O}$-linear too. We claim:

(a) if $\langle V, X\rangle=\langle W, X\rangle$ for every $X \in \mathcal{X}(\mathcal{U})$ then $V=W$;

(b) given any one-form $\omega \in \mathcal{X}^{*}(\mathcal{U})$ there is a uique vector field $V \in \mathcal{X}(\mathcal{U})$ such that $\omega(X)=\langle V, X\rangle$ for every $X \in \mathcal{X}(\mathcal{U})$.

Let $U=V-W$; the nondegeneracy of the metric tensor implies that, if $p \in \mathcal{U}$ and $\left\langle U_{p}, X_{p}\right\rangle=0$ for every $X \in \mathcal{X}(\mathcal{U})$, then $U=0$; this proves (a).

To prove (b), let $\left(z^{1} \ldots z^{N}\right)$ be local coordinates on $\mathcal{U}$.

Then $\omega=\sum_{i=1}^{N} \omega_{i} d z^{i}$; let $\left\{g_{i j}\right\}$ be the representative matrix of $\left.\Lambda\right|_{\mathcal{U}}$ in $\left(z^{1} \ldots z^{N}\right)$ : by nondegeneracy, it admits a holomorphic inverse matrix $\left\{g^{i j}\right\}$ : set now $V=$ $\sum_{j=1}^{N}\left(\sum_{i=1}^{N} g^{i j} \omega_{i}\right) \frac{\partial}{\partial z^{j}}$.

We have $\langle V, X\rangle=\left\langle\sum_{j=1}^{N}\left(\sum_{i=1}^{N} g^{i j} \omega_{i}\right) \frac{\partial}{\partial z^{j}}, \sum_{k=1}^{N} X^{k} \frac{\partial}{\partial z^{k}}\right\rangle=\sum_{i j k} g^{i j} \omega_{i} X^{k} g_{j k}=$ $\sum_{i k} \delta_{k}^{i} X^{k} \omega^{i}=\sum_{k} X^{k} \omega^{k}=\omega(X)$.

The following theorem can be proved exactly as in classical differential geometry.

Theorem 3. Let $\mathcal{U}$ be an open set belonging to a maximal atlas $\mathcal{A}$ for the nondegenerate holomorphic Riemannian manifold $\mathrm{M}$. There exists a unique connexion $D$ on $\mathcal{U}$, called the Levi-Civita connexion, such that:

(D4) $[V, W]=D_{V} W-D_{W} V$;

(D5) $X\langle V, W\rangle=\left\langle D_{X} V, W\right\rangle+\left\langle V, D_{X} W\right\rangle$ for every $X, V, W \in \mathcal{X}(\mathcal{U})$.

Moreover $D$ is characterized by the 'Koszul's formula': $2\left\langle D_{V} W, X\right\rangle=V\langle W, X\rangle+$ $W\langle X, V\rangle-X\langle V, W\rangle-\langle V,[W, X]\rangle+\langle W,[X, V]\rangle+\langle X,[V, W]\rangle$, for every $X, V, W \in \mathcal{X}(\mathcal{U})$. 
If we have to emphasize the open set $\mathcal{U}$ in theorem 3 we shall write $D[\mathcal{U}]$ instead of $D$ : if $\mathcal{U}_{1}, \mathcal{U}_{2} \subset \mathrm{M}$ in a maximal atlas $\mathcal{A}$ for $\mathrm{M}$ are overlapping open sets, then $\mathcal{U}_{1} \bigcap \mathcal{U}_{2}$ is in $\mathcal{A}$ too and $\left.D\left[\mathcal{U}_{1}\right]\right|_{\mathcal{X}\left(\mathcal{U}_{1} \cap \mathcal{U}_{2}\right)}=\left.D\left[\mathcal{U}_{1}\right]\right|_{\mathcal{X}\left(\mathcal{U}_{1} \cap \mathcal{U}_{2}\right)}$, hence we can collect all local definitions of Levi-Civita connexions:

Definition 11. the Levi-Civita connexion (or metric connexion) $D$ of $(\mathrm{M}, \Lambda$ ) is the collection consisting of all the metric connexions $\left\{D\left[\mathcal{U}_{i}\right]\right\}_{i \in I}$ as $\mathcal{U}_{i}$ runs over any maximal atlas $\mathcal{A}=\left(\left\{\mathcal{U}_{i}\right\}\right)_{i \in I}$ on M.

So far we have studied nondegenerate holomorphic Riemannian manifolds: this situation is quite similar to real Riemannian geometry.

Things are different, instead, if we allow metrics to have meromorphic behaviour, or to lower somwhere in their ranks. These metric 'singularities' will be generally supposed to lie in closed hypersurfaces; Levi Civita connexions may still be defined, but, as one could expect, they will turn out to be themselves 'meromorphic'.

Let now $(\mathrm{N}, \Lambda)$ be a meromorphic Riemannian manifold admitting closed hypersurfaces $\mathcal{D}$ and $\mathcal{E}$ such that $\left.\Lambda\right|_{\mathbb{N} \backslash \mathcal{E}}$ is holomorphic and $\left.\Lambda\right|_{(\mathbb{N} \backslash \mathcal{E} \backslash \backslash \mathcal{D}}$ is nondegenerate. Since $\mathbb{N} \backslash \mathcal{E}$ is connected, we have that $(\mathrm{N} \backslash \mathcal{E}) \backslash \mathcal{D},\left.\Lambda\right|_{(\mathrm{N} \backslash \mathcal{E}) \backslash \mathcal{D}}$ is a nondegenerate holomorphic Riemannian manifold admitting, as such, a canonical holomorphic Levi-Civita connexion $D$.

Now, if $p \in \mathcal{D} \cup \mathcal{E}$ and $V, W$ are holomorphic vector fields in a neighbourhood $\mathcal{V}$ of $p$, it will result that we are able to define the vector field $D_{V} W$ on $\mathcal{V} \backslash(\mathcal{D} \cup \mathcal{E})$, and this will be a meromorphic vector field.

Let's state all this more precisely:

Definition 12. Let $Z=\left(z^{1} \cdots z^{m}\right)$ be a coordinate system on an open set $\mathcal{U} \subset \mathrm{N}$ : the Christoffel symbols of $Z$ are those complex valued functions, defined on $\mathcal{U} \backslash(\mathcal{D} \cup \mathcal{E})$ by setting $\Gamma_{i j}^{k}=d z^{k}\left(D_{\frac{\partial}{\partial z^{i}}}\left(\frac{\partial}{\partial z^{j}}\right)\right)$.

Now the representative matrix $\left(g_{i j}\right)$ of $\Lambda$ with respect to the coordinate system $Z$ is holomorphic in $\mathcal{U}$, with nonvanishing determinant function on $\mathcal{U} \backslash(\mathcal{D} \cup \mathcal{E})$; as such it admits a inverse matrix $g^{i j}$, whose coefficients hence result in being $\mathcal{D} \cup \mathcal{E}$-meromorphic functions.

Lemma 6. (a) $D_{\frac{\partial}{\partial z^{i}}}\left(\sum_{j=1}^{m} W^{j} \frac{\partial}{\partial z^{j}}\right)=\sum_{k=1}^{m}\left(\frac{\partial W^{k}}{\partial z^{i}}+\sum_{j=1}^{m} \Gamma_{i j}^{k} W^{j}\right) \frac{\partial}{\partial z^{k}}$ as meromorphic vector fields; (b) $2 \Gamma_{i j}^{k}=\sum_{m=1}^{N} g^{k m}\left(-g_{i j, m}+g_{i m, j}+g_{j m, i}\right)=2 \Gamma_{i j}^{k}$ as meromorphic functions.

Proof. At first note that the operation of associating Christoffel symbols to a coordinate system is compatible with restrictions, in the sense that the Christoffel symbols of the restriction of $Z$ to a smaller open set are its Christoffel symbols restricted to that set. Now, if $p \in \mathcal{U} \bigcap\{n \in \mathrm{N}: \Lambda$ is holomorphic and nondegenerate at $n\}$ and $\mathcal{V}_{p} \subset \mathcal{U}$ is a neighbourhood of $p$, contained in $\mathcal{U}$, we have that $\Lambda$ is holomorphic and nondegenerate in $\mathcal{V}_{p}$ : hence (a): by Koszul's formula we have

$$
2 \sum_{a=1}^{N} \Gamma_{i j}^{a} g_{a m}=2\left\langle D_{\frac{\partial}{\partial z^{i}}} \frac{\partial}{\partial z^{j}}, \frac{\partial}{\partial z^{m}}\right\rangle=\frac{\partial}{\partial z^{i}} g_{j m}+\frac{\partial}{\partial z^{j}} g_{i m}+\frac{\partial}{\partial z^{m}} g_{i j} ;
$$


multiplying both side by $g^{m k}$ and summing over $m$ yields the desired result; (b) follows immediately from (D3) of definition 10. Now the fact that (a) and (b) hold in fact on $\mathcal{U}$ follows by analytical continuation: note that this result does not depend on the choice of $p$.

Proposition 13. For every pair $V, W$ of holomorphic vector fields on the open set $\mathcal{U}$ ( belonging to a maximal atlas) in the meromorphic Riemannian manifold (N, $\Lambda), D_{V} W$ is a well defined vector field, holomorphic on $\mathcal{U} \bigcap\{n \in \mathrm{N}$ : $\Lambda$ is holomorphic and nondegenerate at $n\}$ and may be extended to a meromorphic vector field on $\mathcal{U}$.

Proof. There exist holomorphic functions $\left\{V^{i}\right\},\left\{W^{j}\right\}$ and a coordinate system $Z=$ $\left(z^{1} \ldots . . z^{N}\right)$ on $\mathcal{U}$ such that $V=\sum_{i=1}^{N} V^{i} \frac{\partial}{\partial z^{i}}$ and $W=\sum_{j=1}^{N} W^{i} \frac{\partial}{\partial z^{j}}$. By lemma 6(a),

$$
D_{V} W=\sum_{i=1}^{N} V^{i} D_{\frac{\partial}{\partial z^{i}}}\left(\sum_{j=1}^{N} W^{j} \frac{\partial}{\partial z^{i}}\right)=\sum_{k=1}^{N}\left(\sum_{i, j=1}^{N} V^{i}\left(\frac{\partial W^{k}}{\partial z^{i}}+\Gamma_{i j}^{k} W^{j}\right)\right) \frac{\partial}{\partial z^{k}}:
$$

this is a vector field whose components are meromorphic functions.

Summing up, we yield:

Definition 14. Given a $\mathcal{D}$-degenerate and $\mathcal{E}$-meromorphic Riemannian manifold $(\mathrm{N}, \Lambda)$, with $\mathcal{D}$ and $\mathcal{E}$ closed hypersurfaces in $\mathrm{N}$, the Levi-Civita metric connexion (or meromorphic metric connexion) of $\mathrm{N}$ is the collection consisting of the metric connexions $\left\{D\left[\mathcal{U}_{i} \backslash(\mathcal{D} \bigcup \mathcal{E})\right]\right\}_{i \in I}$ as $\left.\mathcal{U}\right\}_{i}$ runs over any maximal atlas $\mathcal{B}=\left(\{\mathcal{U}\}_{i}\right)_{i \in I}$ on $\mathrm{N}$.

\subsection{Meromorphic parallel translation}

We turn now to study vector fields on paths: an obvious example is the velocity field ( see lemma 4): just as in semi-Riemannian geometry, there is a natural way of defining the rate of change $X^{\prime}$ of a meromorphic vector field $X$ on a path. We study at first paths with values in a nondegenerate holomorphic Riemannian manifold M: let $Q_{\mathrm{M}}=(S, \pi, j, \gamma, \mathrm{M})$ be a path in M, $P$ be the set of branch points of $\pi, r \in S \backslash P$ be a finite-velocity point of $Q_{\mathrm{M}}$. Moreover, let $\mathcal{V} \subset S \backslash P$ be a neighbourhood of $r$ such that $\gamma(\mathcal{V})$ is contained in a local chart in $\mathrm{M}, \mathcal{H}(\mathcal{V})$ be the ring of holomorphic functions on $\mathcal{V}, \mathcal{X}_{\gamma}(\mathcal{V})$ the Lie algebra of holomorphic vector fields over $\gamma$ on $\mathcal{V}$.

Due to the locally nondegenerate holomorphic environment, the following proposition can be proved in quite a classical fashion.

Proposition 15. There exists a unique mapping $\nabla_{\gamma^{\prime}}: \mathcal{X}_{\gamma}(\mathcal{V}) \rightarrow \mathcal{X}_{\gamma}(\mathcal{V})$, called induced covariant derivative on $Q_{\mathrm{M}}$ in $\mathcal{V}$, (or on $\gamma$ in $\mathcal{V}$ ) such that:

$$
\begin{aligned}
& \nabla_{\gamma^{\prime}}\left(a Z_{1}+b Z_{2}\right)=a \nabla_{\gamma^{\prime}} Z_{1}+b \nabla_{\gamma^{\prime}} Z_{2}, \quad a, b \in \mathbb{C} ; \\
& \nabla_{\gamma^{\prime}}(h Z)=\left(\frac{\widetilde{d}}{d z} h\right) Z+h \nabla_{\gamma^{\prime}} Z, \quad h \in \mathcal{H}(\mathcal{V}) ; \\
& \nabla_{\gamma^{\prime}}(V \circ \gamma)(r)=D_{\left.\gamma_{*}\right|_{r}\left(\left.\frac{\widetilde{d}}{d z}\right|_{r}\right)} r \in \mathcal{V},
\end{aligned}
$$


where $V$ is a holomorphic vector field in a neighbourhood of $\gamma(r)$. Moreover,

$$
\frac{\widetilde{d}}{d z}\langle X, Y\rangle=\left\langle\nabla_{\gamma^{\prime}} X, Y\right\rangle+\left\langle X, \nabla_{\gamma^{\prime}} Y\right\rangle \quad X, Y \in \mathcal{X}_{\gamma}(\mathcal{V})
$$

Now let $\mathcal{R}=\left\{\mathcal{V}_{k}\right\}_{k \in K}$ be a maximal atlas for $S \backslash P$; we may assume that, for every $k$, maybe shrinking $\mathcal{V}_{k}, \gamma\left(\mathcal{V}_{k}\right)$ is contained in some local chart $\mathcal{U}_{i}$ in the already introduced atlas $\mathcal{A}$ for $\mathrm{M}$.

By proposition 15, if $\mathcal{V}_{1}$ and $\mathcal{V}_{2}$ are overlapping open sets in $\mathcal{R}, \mathcal{V}_{1} \cap \mathcal{V}_{2} \in \mathcal{R}$ too, and $\left.\nabla_{\gamma^{\prime}}\left[\mathcal{V}_{1}\right]\right|_{\mathcal{V}_{1} \cap \mathcal{V}_{2}}=\left.\nabla_{\gamma^{\prime}}\left[\mathcal{V}_{2}\right]\right|_{\mathcal{V}_{1} \cap \mathcal{V}_{2}}$

Now let's complete $\mathcal{R}$ to an atlas $\mathcal{S}$ for $S$ : keeping into account that the local coordinate expression of the induced covariant derivative is

$$
\nabla_{\gamma^{\prime}} Z=\sum_{k=1}^{m}\left(\frac{\widetilde{d}}{d z} Z^{k}+\sum_{i, j=1}^{m} \Gamma_{i j}^{k} \frac{\widetilde{d}}{d z}\left(u^{i} \circ \gamma\right) Z^{j}\right) \frac{\partial}{\partial u^{k}}
$$

and arguing in the same way as about the meromorphic Levi-Civita connexion, we are able to show that pairs of holomorphic vector fields on $\gamma$ are transormed into $P$-meromorphic vector fields on $\gamma$.

Definition 16. The $P$-meromorphic induced covariant derivative, or the $P$ meromorphic parallel translation on a path $Q_{\mathrm{M}}=(S, \pi, j, \gamma$,$) with set of branch points$ $P$ and taking values in a nondegenerate Riemannian manifold $\mathrm{M}$ is the collection consisting of the induced covariant derivatives $\nabla_{\gamma^{\prime}}\left[\mathcal{V}_{k} \backslash P\right]$ as $\mathcal{V}_{k}$ runs over a maximal atlas $\mathcal{S}=\left(\left\{\mathcal{V}_{k}\right\}\right)_{k \in K}$ on $S$.

Let's turn now to dealing with meromorphic parallel translations induced on a path $Q_{\mathrm{N}}=$ $(T, \varrho, j, \delta)$, in a meromorphic Riemannian manifold $(\mathrm{N}, \Lambda)$ admitting closed hypersurfaces $\mathcal{D}$ and $\mathcal{E}$ such that $\left.\Lambda\right|_{\mathbb{N} \backslash \mathcal{E}}$ is holomorphic and $\left.\Lambda\right|_{(\mathbb{N} \backslash \mathcal{E}) \backslash \mathcal{D}}$ is nondegenerate. We set $\mathcal{F}=\mathcal{D} \bigcup \mathcal{E}$ and restrict our attention to paths starting at metrically ordinary points.

Lemma 7. Set $\mathrm{M}=\mathrm{N} \backslash \mathcal{F}, S=\delta^{-1}(\mathrm{M})$ : then $T \backslash S$ is discrete, hence $S$ is a connected Riemann surface.

Proof. Suppose that there exists a subset $\mathcal{V} \subset T \backslash S$ admitting an accumulation point $t \in \mathcal{V}$ and consider a countable atlas for $\mathcal{B}=\left\{U_{n}\right\}_{n \in \mathbb{N}}$ for $\mathrm{N}$ such that, for every $n$, there exists $\Psi_{n} \in \mathcal{O}\left(\left\{U_{n}\right\}\right)$ such that $U_{n} \bigcap \mathcal{F}=\left\{X \in U_{n}: \Psi_{n}=0\right\}$.

Set $\delta^{-1}\left(U_{n}\right)=T_{n} \subset T$ and suppose, without loss of generality, that $\delta(t) \in U_{0}$.

We have $\left.\Psi_{0} \circ \delta\right|_{\mathcal{V} \cap T_{0}}=0$ and $t \in \mathcal{V} \cap T_{0}$ is an accumulation point of $\mathcal{V} \cap T_{0}$, hence $\left.\Psi_{0} \circ \delta\right|_{T_{0}}=0$ and $\delta\left(T_{0}\right) \subset \mathcal{F}$.

Suppose now that $T_{N} \neq \emptyset$ for some $N$ : we claim that this implies $\delta\left(T_{N}\right) \subset \mathcal{F}$ : to prove the asserted, pick two points $\tau_{0} \in T_{0}$ and $\tau_{n} \in T_{n}$ and two neighbourhoods $T_{0}^{\prime}$, $T_{N}^{\prime}$ of $\tau_{0}$ and $\tau_{n}$ in $T_{0}$ and $T_{n}$ respectively, such that $\left.\varrho\right|_{T_{0}^{\prime}}$ and $\left.\varrho\right|_{T_{N}^{\prime}}$ are biholomorphic functions. Now the function elements $\left(\varrho\left(T_{0}^{\prime}\right), \delta \circ\left(\left.\varrho\right|_{T_{0}^{\prime}}\right)^{-1}\right)$ and $\left(\varrho\left(T_{N}^{\prime}\right), \delta \circ\left(\left.\varrho\right|_{T_{N}^{\prime}}\right)^{-1}\right)$ are connectible, hence there exists a finite chain $\left\{W_{\nu}\right\}_{\nu=0 \ldots L}$ such that $W_{0}=\varrho\left(T_{0}^{\prime}\right), W_{L}=$ $\varrho\left(T_{N}^{\prime}\right), W_{\nu} \cap W_{\nu+1} \neq 0$ for every $\nu$. 
Without loss of generality, we may suppose that each $W_{\nu}$ admits a holomorphic, hence open, immersion $j_{\nu} \rightarrow T$, hence, setting $S_{0}=T_{0}, S_{\lambda}=j_{\lambda}\left(W_{\lambda}\right)$ for $\lambda=1 \ldots L$ and $S_{L+1}=$ $T_{N}$ yields a finite chain of open subsets $\left\{S_{\lambda}\right\}_{\lambda=0 \ldots M}$ of $T$ connecting $T_{0}$ and $T_{N}$.

Let's prove, by induction, that, for every $\lambda, \delta\left(S_{\lambda}\right) \subset \mathcal{F}$.

- At first recall that $\delta\left(S_{0}\right) \subset U_{0} \bigcap \mathcal{F}$ as already proved; suppose now that $\delta\left(S_{k-1}\right) \subset \mathcal{F}$. We have $S_{k-1} \cap S_{k} \neq \emptyset$, hence $\delta\left(S_{k-1}\right) \bigcap \delta\left(S_{k}\right) \neq \emptyset$.

For every $m$ set $\Sigma_{k m}=\delta\left(S_{k-1}\right) \bigcap \delta\left(S_{k}\right) \bigcap U_{m}$ : if $\Sigma_{k m} \neq \emptyset$, then $\Psi_{m} \circ \delta \equiv 0$ on $\delta^{-1}\left(\Sigma_{k m}\right) \bigcap S_{k-1} \bigcap S_{k}$; but $\delta^{-1}\left(\Sigma_{k m}\right) \bigcap S_{k-1} \bigcap S_{k}$ is open in $\delta^{-1}\left(\delta\left(S_{k}\right) \bigcap U_{m}\right) \bigcap S_{k}$, thus $\Psi_{m} \circ \delta \equiv 0$ on $\delta^{-1}\left(\delta\left(S_{k}\right) \bigcap U_{m}\right) \bigcap S_{k}$, that is to say $\delta\left(S_{k}\right) \bigcap U_{m} \subset \mathcal{F}$.

- On the other hand, if $\Sigma_{k m}=\emptyset$, but $\delta\left(S_{k}\right) \cup U_{m} \neq \emptyset$ we claim that $\delta\left(S_{k}\right) \cap U_{m} \subset \mathcal{F}$ as well: proving this requires a further induction: pick a $U_{M}$ such that $\Sigma_{k M} \neq \emptyset$ and a finite chain of open sets $\mathcal{B}^{\prime}=\left\{U_{\mu}^{\prime}\right\}_{\mu=0 \ldots J} \subset \mathcal{B}$ (with $U_{\mu}^{\prime} \cap \delta\left(S_{k}\right) \neq \emptyset$ for each $\mu$ ) connecting $U_{M}$ and $U_{m}$. Since $\Sigma_{k M} \neq \emptyset, \delta\left(S_{k}\right) \bigcap U_{0}^{\prime}=\delta\left(S_{k}\right) \bigcap U_{M} \subset \mathcal{F}$.

Suppose by induction that $\delta\left(S_{k}\right) \bigcap U_{l-1}^{\prime} \subset \mathcal{F}$.

Then $\Psi_{l} \circ \delta \equiv 0$ on $\delta^{-1}\left(\delta\left(S_{k}\right) \cap U_{l-1}^{\prime} \cap U_{l}^{\prime}\right) \cap S_{k}$, hence $\Psi_{l} \circ \delta \equiv 0$ on $\delta^{-1}\left(\delta\left(S_{k}\right) \cap U_{l}^{\prime}\right) \cap S_{k}$ i.e. $\delta\left(S_{k}\right) \cap U_{l}^{\prime} \subset \mathcal{F}$ : this ends the induction and eventually implies $\delta\left(S_{k}\right) \cap U_{m}=$ $\delta\left(S_{k}\right) \cap U_{J}^{\prime} \subset \mathcal{F}$.

Summing up, $\delta\left(S_{k}\right)=\bigcup_{m}\left(\delta\left(S_{k}\right) \cap U_{m}\right) \subset \mathcal{F}$, for each $k$. Hence $\delta\left(T_{N}\right)=\delta\left(S_{M}\right) \subset \mathcal{F}$ and eventually $\delta(T)=\delta\left(\bigcup_{N \in \mathbb{N}} T_{N}\right) \subset \mathcal{F}$, hence $\delta$ cannot start at a point in $\mathrm{N} \backslash \mathcal{F}$.

In the following considerations, there will still hold all notations introduced in preceding lemma: given a path $Q_{\mathrm{N}}=(T, \varrho, j, \delta)$, set $\pi=\left.\varrho\right|_{S}, \gamma=\left.\delta\right|_{S}$ and note that, since $Q_{\mathrm{N}}$ is starting from a metrically ordinary point $m, j$ may be supposed to take values in fact in $S$; since the preceding lemma shows that $S$ is a connected Riemann surface, $Q_{\mathrm{M}}=\left(S, \pi, j,\left.\delta\right|_{S}\right)$ is in fact a path in M, which we call the depolarization of $Q_{\mathrm{N}}$. But M is a nondegenerate holomorphic Riemannian manifold, hence if $P$ is the set of branch points of $\pi$, there is a $P$-meromorphic induced parallel translation on $Q_{\mathrm{M}}$, got following definition 16 and its substratum. Finally, we introduce a maximal atlas $\mathcal{T}$ for $T$ and yield the following:

Definition 17. Let $(\mathrm{N}, \Lambda)$ be a $\mathcal{E}$ - meromorphic and $\mathcal{D}$-degenerate Riemannian manifold, $\mathrm{M}=\mathrm{N} \backslash(\mathcal{D} \cup \mathcal{E}), Q_{\mathrm{N}}=(T \bigcup, \varrho, j, \delta)$ a path: the $\left(P \bigcup \delta^{-1}(\mathcal{D} \bigcup \mathcal{E})\right)$-meromorphic induced covariant derivative on $Q_{\mathrm{N}}$ is the collection consisting of all induced covariant derivatives $\nabla_{\gamma^{\prime}}\left[\mathcal{V}_{k} \cap S\right]$ as $\mathcal{V}_{k}$ runs over a maximal atlas $\mathcal{T}=\left(\left\{\mathcal{V}_{k}\right\}\right)_{k \in K}$ for $T$ and $Q_{\mathrm{M}}=\left(S, \pi, j,\left.\delta\right|_{S}\right)$ is the depolarization of $Q_{\mathrm{N}}$.

\subsection{Geodesics}

Definition 18. A meromorphic (in particular, holomorphic) vector field $Z$ on a path $Q_{\mathrm{M}}=(S, \pi, j, \gamma)$ is parallel provided that $\nabla Z=0$ (as a meromorphic field on $\left.Q_{\mathrm{M}}\right)$.

Definition 19. The acceleration $\aleph\left(Q_{\mathrm{M}}\right)$ of $Q_{\mathrm{M}}$ is the meromorphic field $\nabla\left(V\left(Q_{\mathrm{M}}\right)\right)$ on $Q_{\mathrm{M}}$ yielded by the induced covariant derivative of its velocity field; the speed of a path is the 'amplitude' function of its velocity field: $S\left(Q_{\mathrm{M}}\right)(r)=\left\langle\left.\gamma_{*}\right|_{r}\left(\widetilde{d} \frac{\widetilde{d}}{d z}\right),\left.\gamma_{*}\right|_{r}\left(\frac{\widetilde{d}}{d z}\right)\right\rangle$. This is a meromorphic function. A path is null provided that its speed is zero everywhere.

Definition 20. A geodesic in a meromorphic (in particular, holomorphic) Riemannian manifold is a path whose velocity field is parallel, or, equivalently, one of zero acceleration (see definition 19). A geodesic is null provided that so is as a path. 
The local equations of elements of geodesics $(U, \beta) \ddot{\beta}^{k}+\sum_{i, j=1}^{N} \Gamma_{i j}^{k}(\beta) \dot{\beta}^{i} \dot{\beta}^{j}=0,(k=$ $1 \ldots . . N)$ are a system of $N$ second-order ordinary differential equations in the complex domain, with meromorphic coefficients, in turn equivalent to an autonomous system of $2 N$ first-order equations, hence, as a consequence of the general theory (see theorem 1) we have the following

Theorem 4. For every metrically ordinary point $p \in \mathrm{M}$, every holomorphic tangent vector $V_{p} \in T_{p} \mathrm{M}$ and every $z_{0} \in \mathbb{C}$, there exists a unique germ $\boldsymbol{\beta}_{\boldsymbol{z}_{0}}$ of geodesic such that $\boldsymbol{\beta}_{\boldsymbol{z}_{0}}\left(z_{0}\right)=p$ and $\left.\boldsymbol{\beta}_{\boldsymbol{z}_{0} *}(d / d z)\right|_{z_{0}}=V_{p}$; moreover any analytical continuation of $\boldsymbol{\beta}_{\boldsymbol{z}_{0}}$ is a geodesic.

\section{Completeness theorems}

\subsection{Complex warped products}

In this section we shall be concerned with warped products of Riemann surfaces, each one endowed with some meromorphic metric: in this framework we shall prove a geodesic completeness criterion.

Let now $\mathcal{U}_{i},(i=1 \ldots . N), N \geq 2$ be either a copy of the unit ball in the complex plane, or the complex plane itself, whose coordinate function we shall call $u^{i}$.

Moreover, let each $\mathcal{U}_{i}$ be endowed with a (not everywhere vanishing) meromorphic metric, which we denote by $b_{1}\left(u^{1}\right) d u^{1} \odot d u^{1}$ on $\mathcal{U}_{1}$, or by $f_{i}\left(u^{i}\right) d u^{i} \odot d u^{i}$ if $i \geq 2$, where both $b_{1}$ and the $f_{i}$ 's are nonzero meromorphic functions.

Consider now the meromorphic Riemannian manifold

$$
\mathcal{U}=\mathcal{U}_{1} \times_{a_{2}\left(u^{1}\right)} \mathcal{U}_{2} \times_{a_{3}\left(u^{1}\right)} \mathcal{U}_{3} \times \ldots \ldots . . . \times_{a_{N}\left(u^{1}\right)} \mathcal{U}_{N},
$$

where the $a_{k}$ 's $(k \geq 2)$ are nonzero meromorphic warping functions defined on $\mathcal{U}_{1}$, i.e. depending solely on $u^{1}$.

We could write down the meromorphic metric $\Lambda$ of $\mathcal{U}$ in the form

$$
\Lambda\left(u^{1} \ldots . . u^{N}\right)=b_{1}\left(u^{1}\right) d u^{i} \odot d u^{i}+\sum_{i=2}^{N} a_{i}\left(u^{i}\right) f_{i}\left(u^{i}\right) d u^{i} \odot d u^{i} .
$$

In other words, the matrix of $\Lambda$, with respect to the canonical coordinates of $\mathcal{U}$, inherited from $\mathbb{C}^{N}$, is of the form $\left(g_{i k}\right)=\operatorname{diag}\left(b_{1}\left(u^{1}\right), a_{2}\left(u^{1}\right) f_{2}\left(u^{2}\right), a_{3}\left(u^{1}\right) f_{3}\left(u^{3}\right), \ldots a_{N}\left(u^{1}\right) f_{N}\left(u^{N}\right)\right)$. The following lemma can be proved by easy calculations:

Lemma 8. The meromorphic Levi-Civita connexion induced on $\mathcal{U}$ by $\Lambda$ admits the following Christoffel symbols: $2 \Gamma_{11}^{1}=b_{1}^{\prime}\left(u^{1}\right) / b_{1}\left(u^{1}\right) ; \Gamma_{i j}^{1}=0$ if $i \neq j ; 2 \Gamma_{i i}^{1}=$ $-\left[a_{i}^{\prime}\left(u^{1}\right) f_{i}\left(u^{i}\right)\right] / b_{1}\left(u^{1}\right)$ if $1 \leq i \leq N ; 2 \Gamma_{k k}^{k}=f_{k}^{\prime}\left(u^{k}\right) / f\left(u^{k}\right)$ if $2 \leq k \leq N$ and $2 \Gamma_{i k}^{k}=a_{k}^{\prime}\left(u^{1}\right) / a_{k}\left(u^{1}\right)$ if $i=1$ and $2 \leq k \leq N$. Finally, $\Gamma_{i j}^{k}=0$ otherwise.

As an immediate consequence, we have:

Lemma 9. Each element of geodesic of $(\mathcal{U}, \Lambda)$ satisfies the following system of $N$ ordinary differential equations in the complex domain: 


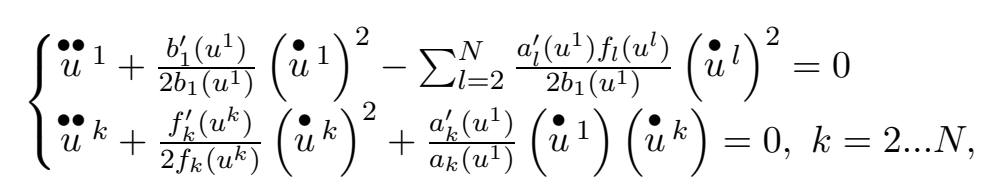

provided that it starts at a metrically ordinary point. Here, and in the following, $u^{k}=$ $u^{k}(z)$.

Lemma 10. The system (5.1) of the differential equations of elements of geodesics $z \mapsto$ $\left(u^{1}(z) \ldots u^{N}(z)\right)$ of $(\mathcal{U}, \Lambda)$ such that the initial values $\left(u^{1}\left(z_{0}\right) \ldots . . u^{N}\left(z_{0}\right), \dot{u}^{1}\left(z_{0}\right) \ldots . . \dot{u}^{N}\left(z_{0}\right)\right)$ of $\gamma$ yield a metrically ordinary point of $(\mathcal{U}, \Lambda) u^{1}$ is not a constant function admits the following first integral:

$$
\begin{array}{r}
\left(\dot{u}^{1}\right)^{2}\left(b_{1}\left(u^{1}\right)\right)=A_{1}-\sum_{l=2}^{N} \frac{A_{l}}{a_{l}\left(u^{1}\right)} \\
\left(\dot{u}^{k}\right)^{2} f_{k}\left(u^{k}\right)\left[a_{k}\left(u^{1}\right)\right]^{2}=A_{k} \quad k=2 \ldots N
\end{array}
$$

Here the $A_{k}$ 's are suitable complex constants.

Proof. Let us prove at first the set of equations (5.3) corresponding to $k=2 \ldots N$.

If $u^{k}$ is a constant function, then $\dot{u}^{k} \equiv 0$ and the k-th equation in (5.3) holds, with $A_{k}=0$.

Otherwise, we could divide the $\mathrm{k}$-th equation in (5.1) by $u^{k}$, this division being lead within the ring of meromorphic functions in a neigbhourhood of $z_{0}$. We get $2 \frac{\ddot{u}^{k}}{\dot{u}^{k}}+$ $\frac{f_{k}^{\prime}\left(u^{k}\right)}{f_{k}\left(u^{k}\right)} \dot{u}^{k}+2 \frac{a_{k}^{\prime}\left(u^{1}\right)}{a_{k}\left(u^{1}\right)} \dot{u}^{1}=0$. Therefore, integrating once, $\left(\dot{u}^{k}\right)^{2} f_{k}\left(u^{k}\right)\left[a_{k}\left(u^{1}\right)\right]^{2}=A_{k}$ where we have set $A_{k}=\left(\dot{u}^{k}\left(z_{0}\right)\right)^{2} f_{k}\left(u^{k}\left(z_{0}\right)\right)\left[a_{k}\left(u^{1}\left(z_{0}\right)\right)\right]^{2}$. Note that $A_{k}$ is a well defined complex number, since $U\left(z_{0}\right)=\left(u^{1}\left(z_{0}\right) \ldots u^{N}\left(z_{0}\right)\right)$ is a metrically ordinary point.

Let us now prove (5.3): we can multiply the first equation of (5.1) by $2 b_{1}\left(u^{1}\right) \dot{u}^{1}$, since this last function is not everywhere vanishing.

We get

$$
2 b_{1}\left(u^{1}\right) \ddot{u}^{1} \ddot{u}^{1}+b_{1}^{\prime}\left(u^{1}\right)\left(\dot{u}^{1}\right)^{3}-\sum_{l=2}^{N} a_{l}^{\prime}\left(u^{1}\right) f_{l}\left(u^{l}\right)\left(\dot{u}^{l}\right)^{2} \ddot{u}^{1}=0
$$

by 5.3 already proved, $\left(\dot{u}^{l}\right)^{2}=A_{l} /\left[f_{l}\left(u^{l}\right)\left[a_{l}\left(u^{1}\right)\right]^{2}\right]$, hence

$$
2 b_{1}\left(u^{1}\right) \dot{u}^{1} \ddot{u}^{1}+b_{1}^{\prime}\left(u^{1}\right)\left(\dot{u}^{1}\right)^{3}-\sum_{l=2}^{N} A_{l} \frac{a_{l}^{\prime}\left(u^{1}\right)}{\left[a_{l}\left(u^{1}\right)\right]^{2}} \dot{u}^{1}=0 .
$$

Integrating once, $b_{1}\left(u^{1}\right)\left(\dot{u}^{1}\right)^{2}+\sum_{l=2}^{N} \frac{A_{l}}{a_{l}\left(u^{1}\right)}=K$ where $K=b_{1}\left(u^{1}\left(z_{0}\right)\right)\left(\dot{u}^{1}\left(z_{0}\right)\right)^{2}+$ $\sum_{l=2}^{N} \frac{A_{l}}{a_{l}\left(u^{1}\left(z_{0}\right)\right)}$. 
Dividing by $b_{1}\left(u^{1}\right)$, keeping into account that $b_{1}\left(u^{1}\left(z_{0}\right)\right) \neq 0$ (due to the metrical ordinariness of the initial point of the geodesic) and eventually setting $A_{1}=K / b_{1}\left(u^{1}\left(z_{0}\right)\right)$ ends the proof.

Lemma 11. Every element of geodesic $z \mapsto\left(u^{1} \ldots u^{N}\right)$ of $(\mathcal{U}, \Lambda)$ such that the initial values $\left(u^{1}\left(z_{0}\right) \ldots . . u^{N}\left(z_{0}\right), \dot{u}^{1}\left(z_{0}\right) \ldots . . \dot{u}^{N}\left(z_{0}\right)\right)$ of $\gamma$ yield a metrically ordinary point of $(\mathcal{U}, \Lambda)$ and $u^{1}$ is a constant function admits the following first integral:

$$
\left(\dot{u}^{k}\right)^{2} f_{k}\left(u^{k}\right)=A_{k} \quad k=2 \ldots N .
$$

Here the $A_{k}$ 's are suitable complex constants.

Proof. If $u^{k}$ is a constant function, then $\dot{u}^{k} \equiv 0$ and the $\mathrm{k}$-th equation in 5.4 holds, with $A_{k}=0$.

Otherwise, we could divide the $\mathrm{k}$-th equation in (5.1) by $u^{k}$, this division being lead within the ring of meromorphic functions in a neigbhourhood of $z_{0}$.

By keeping into account that $\dot{u}^{1} \equiv 0$ we get: $2 \frac{\ddot{u}^{k}}{\dot{u}^{k}}+\frac{f_{k}^{\prime}\left(u^{k}\right)}{f_{k}\left(u^{k}\right)} \dot{u}^{k}=0$. Therefore, integrating once, $\left(\dot{u}^{k}\right)^{2} f_{k}\left(u^{k}\right)=A_{k}$, where we have set $A_{k}=\left(\dot{u}^{k}\left(z_{0}\right)\right)^{2} f_{k}\left(u^{k}\left(z_{0}\right)\right)$. Note that $A_{k}$ is a well defined complex number, since $U\left(z_{0}\right)=\left(u^{1}\left(z_{0}\right) \ldots u^{N}\left(z_{0}\right)\right)$ is a metrically ordinary point: this fact ends the proof.

Remark 21. In the following we shall be concerned with 'extracting square roots' of nonvanishing elements, or germs, of holomorphic functions at some points in the complex plane: more precisely, let $(U, \Psi)$ be a never vanishing HFE: then there exist two HFE's $\left(U, \Xi_{1}\right)$ and $\left(U, \Xi_{2}\right)$ such that $\Xi_{1}^{2}=\Psi$ and $\Xi_{2}^{2}=\Psi$ on $U$ : the Riemann surfaces of $\left(U, \Xi_{1}\right)$ and $\left(U, \Xi_{2}\right)$ are isomorphic, since either the Riemann surface $(R, p, i, \widetilde{U})$ of $(U, \Psi)$ is such that $\widetilde{U}$ is never vanishing, nor has it got any poles; then the Riemann surfaces of $\left(U, \Xi_{1}\right)$, $\left(U, \Xi_{2}\right)$ and $(U, \Psi)$ are all isomorphic, or the Riemann surface $(R, p, i, \widetilde{U})$ of $(U, \Psi)$ is such that there exists some point $p \in R$ such that $\widetilde{U}(p)=0$ or such that $\widetilde{U}$ has a pole in $p$ : then the function elements $\left(U, \Xi_{1}\right)$ and $\left(U, \Xi_{2}\right)$ are connectible, hence their Riemann surfaces are again isomorphic. The same argument could be applied without changes to the Riemann surfaces of the HFE's $\left(U, \int \Xi_{1}\right)$ and $\left(U, \int \Xi_{2}\right)$.

Definition 22. A meromorphic warped product

$$
\mathcal{U}=\mathcal{U}_{1} \times_{a_{2}\left(u^{1}\right)} \mathcal{U}_{2} \times_{a_{3}\left(u^{1}\right)} \mathcal{U}_{3} \times \ldots \ldots \times_{a_{N}\left(u^{1}\right)} \mathcal{U}_{N}
$$

of complex planes or one-dimensional unit balls with metric

$$
\Lambda\left(u^{1} \ldots . . u^{N}\right)=b_{1}\left(u^{1}\right) d u^{i} \odot d u^{i}+\sum_{i=2}^{N} a_{i}\left(u^{i}\right) f_{i}\left(u^{i}\right) d u^{i} \odot d u^{i},
$$

where $b_{1}$, the $a_{k}$ 's and the $f_{k}$ 's are nonzero meromorphic functions is coercive provided that, for every metrically ordinary point $X_{0}=\left(x_{0}^{1} \ldots x_{0}^{N}\right)$ and 
- for every n-tuple $\left(A_{1} \ldots A_{N}\right) \in \mathbb{C}^{N}$ such that $b_{1}\left(x_{0}^{1}\right) \neq 0$ and $A_{1}-\sum_{l=2}^{N} \frac{A_{l}}{a_{l}\left(x_{0}^{1}\right)} \neq 0$ and for each one of the two HFG's $\aleph_{1}$ and $\aleph_{2}$ such that

$$
\left(\aleph_{i}\right)^{2}=\left[\frac{1}{b_{1}}\left(A_{1}-\sum_{l=2}^{N} \frac{A_{l}}{a_{l}}\right)\right]_{x_{0}^{1}} i=1,2,
$$

the Riemann surface $\left(S_{1}, \pi_{1}, j_{1}, \Phi_{1}, \mathcal{U}\right)$ of both the HFG's (see remark 21)

$$
\left[\int_{x_{0}}^{u^{1}} \frac{d \eta}{\aleph_{i}(\eta)}\right]_{x_{0}^{1}} i=1,2
$$

is such that $\mathbb{C} \backslash \Phi_{1}\left(S_{1}\right)$ is a finite set;

- for each $k, 2 \leq k \leq N$ and for each one of the two HFG's $\phi_{k 1}$ and $\phi_{k 2}$ such that

$$
\left(\phi_{k i}\right)^{2}=\left[f_{k}\right]_{x_{0}^{1}}, \quad i=1,2
$$

the Riemann surface $\left(S_{k}, \pi_{k}, j_{k}, \Phi_{k}, \mathcal{U}\right)$ of both the HFG's (see remark 21)

$$
\left[\int_{x_{0}^{1}}^{u^{k}} \phi_{k i}(\eta) d \eta\right]_{x_{0}^{1}} i=1,2
$$

is such that $\mathbb{C} \backslash \Phi_{k}\left(S_{k}\right)$ is a finite set.

Remark 23. Definition 22 may be checked for just one metrically ordinary point $X_{0}$ : this is proved in lemma 12; moreover, we may assume, without loss of generality $X_{0}=0$ : were not, we could carry it into 0 by applying an automorphism of $\mathcal{U}$, that is to say a direct product of automorphisms of the unit ball or of the complex plane, according to the nature of each $\mathcal{U}_{i}$. Then a simple pullback procedure would yield back the initial situation: in the following we shall understand this choice.

In the following lemma we shall use the 'square root' symbol in the meaning of definition 22, or remark 21: in other words, given a HFG, which is not vanishing at some point, it should denote any one of the two HFG's yielding it back when squared.

Lemma 12. For every metrically ordinary point $\left(\xi^{1} \ldots \xi^{N}\right)$ of $\mathcal{U}$ and every $n$-tuple $\left(A_{1} \ldots A_{N}\right) \in \mathbb{C}^{N}$ such that $b_{1}\left(x_{0}^{1}\right) \neq 0, A_{1}-\sum_{l=2}^{N} \frac{A_{l}}{a_{l}\left(x_{0}^{1}\right)} \neq 0, \quad b_{1}\left(\xi^{1}\right) \neq 0$ and $A_{1}-\sum_{l=2}^{N} \frac{A_{l}}{a_{l}\left(\xi^{1}\right)} \neq 0$, set $\Psi(\eta):=A_{1}-\sum_{l=2}^{N} \frac{A_{l}}{a_{l}(\eta)}$ : then the Riemann surfaces of the HFG's $\int_{\xi_{1}}^{u^{1}} \sqrt{b_{1}(\eta) / \Psi(\eta)} d \eta \quad$ at $\xi_{1}$ and $\int_{0}^{u^{1}} \sqrt{b_{1}(\eta) / \Psi(\eta)} d \eta \quad$ at 0 are isomorphic: moreover so are, for each $k$, those of $\int_{\xi_{k}}^{u^{k}} \sqrt{f_{k}(\eta) d \eta}$ at $\xi_{k}$ and $\int_{0}^{u^{k}} \sqrt{f_{k}(\eta) d \eta}$ at 0 .

Proof. The statement easily follows from the fact that those germs are connectible.

Here is the main result concerning warped products of Riemann surfaces: 
Theorem 5. A meromorphic warped product

$$
\mathcal{U}=\mathcal{U}_{1} \times_{a_{2}\left(u^{1}\right)} \mathcal{U}_{2} \times_{a_{3}\left(u^{1}\right)} \mathcal{U}_{3} \times \ldots \ldots \times_{a_{N}\left(u^{1}\right)} \mathcal{U}_{N}
$$

of complex planes or one-dimensional unit balls with metric

$$
\Lambda\left(u^{1} \ldots . . u^{N}\right)=b_{1}\left(u^{1}\right) d u^{1} \odot d u^{1}+\sum_{i=2}^{N} a_{i}\left(u^{1}\right) f_{i}\left(u^{i}\right) d u^{i} \odot d u^{i}
$$

is geodesically complete if and only if it is coercive.

Proof. a) Suppose that $\mathcal{U}$ is coercive and that $U$, defined by $z \mapsto\left(u^{1} \ldots u^{N}\right)$, is an element of geodesic, defined in a neighbourhood of 0 in the complex plane and such that $\left(u^{1}(0) \ldots u^{N}(0)\right)$ is a metrically ordinary point; moreover, let $\left(\dot{u}^{1}(0) \ldots \dot{u}^{N}(0)\right)$ be the initial velocity of $U$.

Suppose at first that $z \mapsto u^{1}$ is a constant function (hence $\left.\dot{u}^{1}(0)=0\right)$ : then, by lemma 11 , the equations of $U$ are

$$
\left(\dot{u}^{k}\right)^{2} f_{k}\left(u^{k}\right)=A_{k} \quad k=2 \ldots N
$$

where the $A_{k}$ 's are suitable complex constants; here $u^{1} \equiv A_{1}$.

Now the Riemann surface of the HFE $z \mapsto u^{1}$ is trivially isomorphic to $\left(\mathbb{C}, \boldsymbol{i d}, \boldsymbol{i d}, A_{1}\right)$; if $A_{k}=0$ the Riemann surface of $z \mapsto u^{k}$ is isomorphic to $(\mathbb{C}, \boldsymbol{i d}, \boldsymbol{i d}, A)$ for some complex constant $A$; if $A_{k} \neq 0$ we could rewrite the k-th equation of (5.7) in the form:

$$
\frac{1}{B_{k}} \int_{u^{k}(0)}^{u^{k}} \phi(\eta) d \eta=z
$$

where $\phi_{k}^{2}=f_{k}$ and $B_{k}^{2}=A_{k}$, the choice of $\phi_{k}$ and $B_{k}$ being made in such a way that $\dot{u}^{k}(0)=\frac{B_{k}}{\phi_{k}(0)}$.

By hypothesis, the Riemann surface $\left(S_{k}, \pi_{k}, j_{k}, \Phi_{k}\right)$ of the HFG $\int_{0}^{u^{k}} \phi_{k} d \eta$ at 0 is such that $\mathbb{C} \backslash \Phi_{1}\left(S_{1}\right)$ is a finite set; by lemma 12 the Riemann surface of the HFG $\int_{u^{k}(0)}^{u^{k}} \phi_{k} d \eta$ at $u^{k}(0)$ is isomorphic to $\left(S_{k}, \pi_{k}, j_{k}, \Phi_{k}\right)$; but, by (5.8), the germs $\boldsymbol{u}_{z=0}^{k}$ and $\int_{u^{k}(0)}^{u^{k}} \phi_{k} d \eta$ at $u^{k}(0)$ are each one inverse of the other; hence, by lemma 2 the Riemann surface of $\boldsymbol{u}_{z=0}^{k}$ is complete; this eventually implies that the Riemann surface of the element $z \mapsto\left(u^{1} \ldots u^{N}\right)$ is complete too: this fact ends the proof of a) in the case that $u^{1}$ is a constant function.

On the other side, suppose that $u^{1}$ is not a constant function: then, by lemma 10, the equations of $U$ are

$$
\left\{\begin{array}{l}
\left(\dot{u}^{1}\right)^{2}\left(b_{1}\left(u^{1}\right)\right)=A_{1}-\sum_{l=2}^{N} \frac{A_{l}}{a_{l}\left(u^{1}\right)} \\
\left(\dot{u}^{k}\right)^{2} f_{k}\left(u^{k}\right)\left[a_{k}\left(u^{1}\right)\right]^{2}=A_{k} \quad k=2 \ldots N .
\end{array}\right.
$$


for suitable complex constants $A_{1} \ldots A_{N}$.

Consider now the germ $z \mapsto u^{1}$ in $z=0$ : rewrite the first equation of (5.9) in the form:

$$
\int_{u^{1}(0)}^{u^{1}} \frac{d \eta}{\aleph(\eta)_{u^{1}(0)}}=z
$$

where $\left(\boldsymbol{\aleph}(\eta)_{u^{1}(0)}\right)^{2}=\left[A_{1}-\sum_{l=2}^{N} A_{l} / a_{l}(\eta)\right] / b_{1}(\eta)$ in a neighbourhood of $z=0$, the choice of the square root $\boldsymbol{\aleph}_{k}$ being made in such a way that $\boldsymbol{\aleph}_{u^{1}(0)}\left(u^{1}(0)\right)=1 / \dot{u}^{1}(0)$.

Denote now by $\boldsymbol{\aleph}_{u=0}$ the HFG defined by setting

$$
\left(\boldsymbol{\aleph}_{0}\right)^{2}=\left[\frac{1}{b_{1}}\left(A_{1}-\sum_{l=2}^{N} \frac{A_{l}}{a_{l}}\right)\right]_{0}
$$

the choice of the 'square root' $\aleph_{0}$ being arbitrary.

By hypothesis, the Riemann surface $\left(S_{1}, \pi_{1}, j_{1}, \Phi_{1}\right)$ of the HFG $\int_{0}^{u^{1}} 1 / \boldsymbol{\aleph}_{0}$ at 0 is such that $\mathbb{C} \backslash \Phi_{1}\left(S_{1}\right)$ is a finite set.

By lemma 12 the Riemann surfaces of $\int_{0}^{u^{1}} 1 / \aleph_{0}$ (at 0$)$ and of $\int_{u_{0}^{1}}^{u^{1}} 1 / \aleph_{0}\left(\right.$ at $\left.u_{0}^{1}\right)$ are both isomorphic to $\left(S_{1}, \pi_{1}, j_{1}, \Phi_{1}\right)$; but, by (5.8), the germs $\boldsymbol{u}_{z=0}^{1}$ and $\int_{0}^{u^{1}} 1 / \boldsymbol{\aleph}_{0}\left(\right.$ at $\left.u^{1}(0)\right)$ are each one inverse of the other; hence, by lemma 2 the Riemann surface of $\boldsymbol{u}_{z=0}^{1}$ is complete.

Let now $2 \leq k \leq N$ : if $A_{k}=0$ the Riemann surface of $z \mapsto u^{k}$ is isomorphic to $(\mathbb{C}, \boldsymbol{i d}, \boldsymbol{i d}, A)$ for some complex constant $A$; if $A_{k} \neq 0$ we could rewrite the k-th equation of (5.9) in the form:

$$
\int_{u^{k}(0)}^{u^{k}} \phi(\eta) d \eta=\int_{0}^{z} \frac{B_{k} d z}{a_{k}\left(u^{1}\right)}
$$

where $\phi_{k}^{2}=f_{k}$ and $B_{k}^{2}=A_{k}$, the choice of $\phi_{k}$ and $B_{k}$ being made in such a way that $\dot{u}^{k}(0) \phi\left(u^{k}(0)\right) a_{k}\left(u^{1}\right)=B_{k}$.

Denote now by $\left[\varphi_{k}\right]_{u^{k}=0}$ the HFG defined by setting $\left[\varphi_{k}\right]_{u^{k}=0}^{2}=\left[f_{k}\right]_{u^{k}=0}$, the choice of the "square root" $\left[\varphi_{k}\right]_{u^{k}=0}$ being arbitrary.

By hypothesis, the Riemann surface $\left(S_{k}, \pi_{k}, j_{k}, \Phi_{k}\right)$ of the HFG $\int_{0}^{u^{k}} \varphi_{k}$ (at 0 ) is such that $\mathbb{C} \backslash \Phi_{1}\left(S_{1}\right)$ is a finite set; moreover, by lemma 12 the Riemann surfaces of the HFG $\int_{u^{k}(0)}^{u^{k}} \phi_{k} d \eta\left(\right.$ at $\left.u^{k}(0)\right)$ is isomorphic to $\left(S_{k}, \pi_{k}, j_{k}, \Phi_{k}\right)$; but, by (5.11) the germs $\left[z \rightarrow \boldsymbol{u}^{k}\right]_{z=0}, \int_{u^{k}(0)}^{u^{k}} \phi_{k} d \eta\left(\right.$ at $u^{k}(0)$ and $z \rightarrow \int_{0}^{z} \frac{B_{k}}{a_{k}\left(u^{1}(\zeta)\right)} d \zeta($ at $z=0)$ satisfy, in the above order, the hypotheses of lemma 2 ; moreover, the Riemann surface with logarithmic singularities of $\int_{u^{k}(0)}^{u^{k}} \phi_{k} d \eta\left(\right.$ at $\left.u^{k}(0)\right)$ is complete, since the one of $\left[\phi_{k}\right]_{u^{k}(0)}$ is complete without logarithmic singularities. 
Therefore the Riemann surface with logarithmic singularities of $\boldsymbol{u}_{z=0}^{k}$ is complete; this eventually implies that the Riemann surface with logarithmic singularities of the element $z \mapsto\left(u^{1} \ldots u^{N}\right)$, is complete too: this fact ends the proof of a).

Vice versa, suppose that $\mathcal{U}=\mathcal{U}_{1} \times_{a_{2}\left(u^{1}\right)} \mathcal{U}_{2} \times_{a_{3}\left(u^{1}\right)} \mathcal{U}_{3} \times \ldots \ldots . . \times_{a_{N}\left(u^{1}\right)} \mathcal{U}_{N}$ is not coercive: then either there exists a complex n-tuple $\left(A_{1} \ldots A_{N}\right) \in \mathbb{C}^{N}$ such that $b_{1}\left(x_{0}^{1}\right) \neq 0, A_{1}-$ $\sum_{l=2}^{N} \frac{A_{l}}{a_{l}\left(x_{0}^{1}\right)} \neq 0$ and for each one of the two HFG's $\aleph_{1}$ and $\aleph_{2}$ such that

$$
\left(\aleph_{i}\right)^{2}=\left[\frac{1}{b_{1}}\left(A_{1}-\sum_{l=2}^{N} \frac{A_{l}}{a_{l}}\right)\right]_{0} \quad i=1,2
$$

the Riemann surface $\left(S_{1}, \pi_{1}, j_{1}, \Phi_{1}\right)$ of both the HFG's (see remark 21) $\int_{x_{0}}^{u^{1}} \frac{d \eta}{\boldsymbol{\aleph}_{i}(\eta)}$ ( at $\left.x_{0}^{1}(i=1,2)\right)$ is such that $\mathbb{C} \backslash \Phi_{1}\left(S_{1}\right)$ is an infinite set; or there exists $k, 2 \leq k \leq N$ such that, for each one of the two HFG's $\left[\phi_{k 1}\right]_{0}$ and $\left[\phi_{k 2}\right]_{0}$ such that $\left[\phi_{k i}\right]_{0}=\left[f_{k}\right]_{0},(i=(1,2))$ the Riemann surface $\left(S_{k}, \pi_{k}, j_{k}, \Phi_{k}\right)$ of both the HFG's (see remark 21) $\left[\int_{0}^{u^{k}} \phi_{k} i(\eta) d \eta\right]_{0} i=$ 1,2 is such that $\mathbb{C} \backslash \Phi_{1}\left(S_{1}\right)$ is an infinite set.

In the first case the geodesic element $z \mapsto U=\left(u^{1} \ldots u^{N}\right)$ starting from 0 with velocity $\left(L_{1} \ldots L_{N}\right)$, such that

$$
L_{1}^{2}=\frac{1}{b_{1}(0)}\left(A_{1}-\sum_{l=2}^{N} \frac{A_{l}}{a_{l}(0)}\right), L_{k}^{2}=\frac{A_{k}}{f_{k}(0) a_{k}(0)}, k=2 \ldots N
$$

satisfies the equation $\int_{0}^{u^{1}} \frac{d \eta}{\aleph_{i}(\eta)}=z, i=1,2$; by lemma 2 , this fact implies that $\left[z \mapsto u^{1}\right]_{0}$ has an incomplete Riemann surface, hence the same holds about $z \mapsto U$ too.

Consider now the second case: first construct a geodesic element $z \mapsto U=\left(0 \ldots u^{k} \ldots 0\right)$, with all components which have to be constant functions except $u^{k}, k \geq 2$ (this element is easily seen to exist).

Now recall lemma 11 to conclude that $z \mapsto u^{k}$ satisfies, in a neighbourhood of $z=0$ the equation $\frac{1}{C_{k}} \int_{0}^{u^{k}} \phi_{k i}(\eta) d \eta=z$, for a suitable complex constant $A_{k}$; therefore its Riemann surface is incomplete by lemma 2 ; this fact ends the proof.

Definition 24. Let $\mathcal{U}$ and $\mathcal{V}$ be meromorphic warped products of complex planes and unit balls; $\mathcal{U}$ and $\mathcal{V}$ are directly biholomorphic provided that they are biholomorphic under a direct product of biholomorphic functions between each $\mathcal{U}_{i}$ and each $\mathcal{V}_{i}$.

Remark 25. Definition 22 is invariant under direct biholomorphism (see definition 24): in other words, if $\mathcal{U}$ and $\mathcal{V}$ are directly biholomorphic, then $\mathcal{U}$ is coercive if and only $\mathcal{V}$ is too: this is a simple consequence of 'changing variable' in integrals 5.5 and 5.6.

Therefore, we could yield the following

Definition 26. An equivalence class $[\mathcal{U}]$ of meromorphic warped products of complex planes and unit balls, consisting of mutually directly (see definition 24 ) biholomorphic elements is coercive provided that any one of its representatives is coercive. 
Our goal is now to extend definitions 22 and 26 to warped products containg some $\mathbb{P}^{1}$ 's among their factors.

Keeping into account remark 25, this could be readiliy pursued: indeed, consider a meromorphic warped product

$$
\mathcal{U}=\mathcal{U}_{1} \times_{a_{2}\left(u^{1}\right)} \mathcal{U}_{2} \times_{a_{3}\left(u^{1}\right)} \mathcal{U}_{3} \times \ldots \ldots \times_{a_{N}\left(u^{1}\right)} \mathcal{U}_{N}
$$

of Riemann spheres, complex planes or one-dimensional unit balls with metric

$$
\Lambda\left(u^{1} \ldots . . u^{N}\right)=b_{1}\left(u^{1}\right) d u^{i} \odot d u^{i}+\sum_{i=2}^{N} a_{i}\left(u^{i}\right) f_{i}\left(u^{i}\right) d u^{i} \odot d u^{i} .
$$

Let $L \subset\{1 \ldots N\}$ be the set of indices such that $\mathcal{U}_{l} \simeq \mathbb{P}^{1}$ for each $l \in L$.

Definition 27. Let $Y=\left(y^{1} \ldots y^{N}\right) \in \mathcal{U}$ : then $(Y, L)$ is a principal multipole of $\mathcal{U}$ provided that $b_{1}\left(y^{1}\right)=\infty$ and $f_{l}\left(y^{l}\right)=\infty$ for each $l \in L \backslash\{1\}$.

Definition 28. A meromorphic warped product

$$
\mathcal{U}=\mathcal{U}_{1} \times_{a_{2}\left(u^{1}\right)} \mathcal{U}_{2} \times_{a_{3}\left(u^{1}\right)} \mathcal{U}_{3} \times \ldots \ldots \times_{a_{N}\left(u^{1}\right)} \mathcal{U}_{N}
$$

of Riemann spheres, complex planes or one-dimensional unit balls with metric is partially projective if some one of its factors is biholomorphic to the Riemann sphere $\mathbb{P}^{1}$.

Definition 29. A partially projective warped product $\mathcal{U}=\prod_{i=1}^{N} \mathcal{U}_{i}$ is coercive in opposition to the principal multipole $(Y, L)$ if, set $\mathcal{W}_{i}=\mathcal{U}_{i}$ if $i \notin L, \mathcal{W}_{i}=\mathcal{U}_{i} \backslash\left\{y^{i}\right\}$ ifi $\in L$, then $\prod_{i=1}^{N} \mathcal{W}_{i}$ is coercive in the sense of definition 26 , that is to say, belongs to a coercive equivalence class with respect to direct biholomorphicity.

\subsection{Warped product of Riemann surfaces}

Consider now a warped product of Riemann surfaces

$$
\mathcal{S}=\mathcal{S}_{1} \times{ }_{a_{2}} \mathcal{S}_{2} \times{ }_{a_{3}} \mathcal{S}_{3} \times \ldots \ldots \times \times_{a_{N}} \mathcal{S}_{N}
$$

where each $\mathcal{S}_{i}$ is endowed with meromorphic metric $\lambda_{i}$ : $\mathcal{S}$ 's metric $\Lambda$ is defined by setting

$$
\Lambda=\lambda_{1}+\sum_{k=2}^{N} a_{k} \lambda_{k}
$$

where each $a_{k}$ is a meromorphic function on $\mathcal{S}_{i}$.

Theorem 6. $\mathcal{S}$ admits universal covering $\Psi: \mathcal{U} \rightarrow \mathcal{S}$, where $\mathcal{U}$ is a direct product of Riemann spheres, complex planes or one-dimensional unit balls: this universal covering is unique up to direct biholomorphisms.

Proof. This is a simple consequence of Riemann's uniformization theorem. 
Now $\mathcal{U}$ could be endowed with the pull-back meromorphic metric $\Psi^{*} \Lambda$, hence $\mathcal{U}$ itself results in a meromorphic warped product.

Definition 30. The manifold $\mathcal{S}$ is totally unelliptic provided that none of the $\mathcal{S}_{i}$ is elliptic; $L$-elliptic provided that there exists a nonempty set of indices $L$ such that $\mathcal{S}_{l}$ is elliptic if and only if $l \in L$.

Definition 31. Let $\mathcal{S}$ be a $L$-elliptic warped product, with universal covering $\Psi: \mathcal{U} \rightarrow \mathcal{S}$ : then $(Z, L)$ is a principal multipole for $\mathcal{S}$ provided that $Z \in \mathcal{S}$ and each $Y \in \Psi^{-1}(Z)$ is a principal multipole for $\mathcal{U}$.

Definition 32. A totally unelliptic warped product of Riemann surfaces is coercive provided that its universal covering is coercive in the sense of definition 26. A $L$-elliptic warped product of Riemann surfaces is coercive in opposition to the principal multipole $(Z, L)$ provided that its universal covering $\mathcal{U}$ is coercive in opposition to each principal multipole $(Y, L)$ as $Y$ runs over $\Psi^{-1}(Z)$.

Theorem 7. A totally unelliptic warped product of Riemann surfaces $\mathcal{S}$ is geodesically complete if and only if it is coercive.

Proof. Let $\Psi: \mathcal{U} \rightarrow \mathcal{S}$ be the universal covering of $\mathcal{S}$ : by definition $30 \mathcal{U}$ is coercive, hence geodesically complete by theorem 5 .

Let now $\gamma$ be a germ of geodesic in $\mathcal{S}$, starting at a metrically ordinary point: since $\Psi$ is a local isometry, there exists a germ $\boldsymbol{\beta}$ of geodesic in $\mathcal{U}$, starting at a metrically ordinary point, such that $\boldsymbol{\gamma}=\Psi \circ \boldsymbol{\beta}$.

By definition of completeness, the Riemann surface with logarithmic singularities $(\Sigma, \pi, j, B, \mathcal{U})$ of $\boldsymbol{\beta}$ is such that $\mathbb{C} \backslash \pi(\Sigma)$ is a finite set; moreover, $(\Sigma, \pi, j, \Psi \circ B, \mathcal{S})$ is an analytical continuation, with logarithmic singularities, of $\gamma$.

This proves that, if $(\widetilde{\Sigma}, \widetilde{\pi}, \widetilde{\mathrm{J}}, G, \mathcal{S})$ is the Riemann surface with logarithmic singularities of $\gamma$, then $\mathbb{P}^{1} \backslash \tilde{\pi}(\widetilde{\Sigma})$ is a finite set too, hence $\mathcal{S}$ is geodesically complete.

On the other side, if $\mathcal{S}$ admits an incomplete germ of geodesic $\gamma$, starting at a metrically ordinary point, then there exists an incomplete germ of geodesic $\boldsymbol{\beta}$ in $\mathcal{U}$, starting at a metrically ordinary point, such that $\gamma=\Psi \circ \boldsymbol{\beta}$; this means by theorem 5 , that $\mathcal{U}$ is not coercive; eventually, by definition $30, \mathcal{S}$ is not coercive: this fact ends the proof.

Theorem 8. A L-elliptic warped product of Riemann surfaces $\mathcal{S}$ is geodesically complete if and only if) it is coercive in opposition to some principal multipole.

Proof. Suppose that $\mathcal{S}$ is coercive in opposition to some principal multipole $(Z, L)$ : then, by theorem $7, \mathcal{S}$ is coercive in opposition to $(Z, L)$ if and only if $\mathcal{S} \backslash Z$ is geodesically complete; since $Z$ is not metrically ordinary, $\mathcal{S}$ is geodesically complete.

On the other hand, suppose that $\mathcal{S}$ admits an incomplete geodesic $(\Sigma, \pi, j, \gamma, \mathcal{S})$ : let $(Z, L)$ be a principal multipole of $\mathcal{S}$ wich is known to exist; set $R=\gamma^{-1}(\mathcal{S} \backslash Z) \subset \Sigma$.

Now $\left(R,\left.\pi\right|_{R}, j,\left.\gamma\right|_{R}, \mathcal{S} \backslash Z\right)$ is an incomplete geodesic of $\mathcal{S} \backslash Z$ : this fact implies that $\mathcal{S} \backslash Z$ is not geodesically complete, hence it is not coercive, that is to say, $\mathcal{S}$ is not coercive in opposition to $(Z, L)$.

The arbitrariness of $Z$ allows us to conclude the proof. 


\subsubsection{Examples}

We shall now show a wide class of warped products sharing all characteristics defining coercivity: they will hence result in being geodesically complete.

We recall, without proof, the following results from the theory of meromorphic functions (see $[\mathrm{NEV}]$ or $[\mathrm{HAY}])$ :

Theorem 9. A meromorphic function in the complex plane takes all $\mathbb{P}^{1}$ 's values but at most two ones; a meromorphic function in the unit disc, whose characteristic function $T$ is such that the ratio $T(r) / \log (1-r)$ is unlimited as $r \rightarrow 1$, takes all $\mathbb{P}^{1}$ 's values but at most two ones.

In the following we shall need some technicalities from integral calculus, hence we state:

Proposition 33. If $\Delta:=b^{2}-4 a c$ then $\left[\int \frac{d \eta}{\sqrt{a \eta^{2}+b \eta+c}}\right]_{0}$ equals one and only one (up to additive constants) of the following expressions, in a neighbourhood of 0 :

(A)

$$
\left\{\begin{array}{l}
{\left[\frac{1}{\sqrt{a}} \log \left(\eta+\frac{b}{2 a}+\sqrt{\eta^{2}+\frac{b}{a} \eta+\frac{c}{a}}\right)\right]_{0}} \\
\text { the same branch of } \sqrt{ }, \text { any branch of } \log \\
\text { if } a \neq 0 \text { and } \Delta \neq 0
\end{array}\right.
$$

(C) $\left\{\begin{array}{l}{\left[\frac{2}{b} \sqrt{b \eta+c}\right]_{0}} \\ \text { the same branch of } \sqrt{ } \\ \text { if } a=0 \text { and } b \neq 0\end{array}\right.$
(D) $\left\{\begin{array}{l}{[\eta / \sqrt{c}]_{0}} \\ \text { the same branch of } \sqrt{ } \\ \text { if } a=b=0\end{array}\right.$

(B) $\left\{\begin{array}{l}{\left[\frac{1}{\sqrt{a}} \log \left(\eta+\frac{b}{2 a}\right)\right]_{0}} \\ \text { any branch of } \log \\ \text { if } a \neq 0 \text { and } \Delta=0\end{array}\right.$

Let now $h, f_{2} \ldots f_{N}$ be meromorphic functions on $\mathbb{C}$ and $P_{2} \ldots P_{N}$ polynomials of degree at most two. Consider on $\mathbb{C}^{N}$ the meromorphic metric

$$
\Lambda\left(u^{1} \ldots u^{N}\right)=\left[h^{\prime}\left(u^{1}\right)\right]^{2} d u^{1} \odot d u^{1}+\sum_{k=2}^{N} \frac{\left[f_{k}\left(u^{k}\right)\right]^{2}}{P_{k}\left(h\left(u^{1}\right)\right)} d u^{k} \odot d u^{k} .
$$

Theorem 10. $\left(\mathbb{C}^{N}, \Lambda\right)$ is coercive (hence geodesically complete).

Proof. For every n-tuple $\left(A_{1} \ldots A_{N}\right) \in \mathbb{C}^{N}$ such that $h^{\prime}(0) \neq 0$ and $A_{1}-\sum_{l=2}^{N} A_{l} P_{l}(0) \neq 0$, set $\Psi(x)=A_{1}-\sum_{l=2}^{N} A_{l} P_{l}(x)$. There holds $\int_{0}^{u^{1}}(\Psi \circ h(\eta))^{-1 / 2} h^{\prime}(\eta) d \eta=\Phi\left(h\left(u^{1}\right)\right)$, where $\Phi$ is one (depending on the constants $A_{1} \ldots A_{N}$ ) of the HFG's on the right hand member of proposition 33. This fact shows that the maximal analytical continuation of $u^{1} \rightarrow \Phi\left(h\left(u^{1}\right)\right)$ takes all $\mathbb{P}^{1}$ 's values but a finite number, because so does the meromorphic function $h$ (see theorem 9).

Moreover, for each $k, 2 \leq k \leq N$, each one of the two HFG's $\pm\left[f_{k}\right]_{0}$, could be continuated to $\pm f_{k}$ which, by theorem 9 , takes all values but at most two ones. 
Remark 34. Extending the validity of preceding example to the partially projective case is straightforward.

Let now $S_{i}, i=1 . . N$ be Riemann surfaces, which we suppose for simplicity to be parabolic or hyperbolic, $p_{i}: \mathcal{U}_{i} \rightarrow S_{i}$ their universal covering, where each $\mathcal{U}_{i}$ is isomorphic either to the unit disc or to the complex plane; finally, let $\phi_{i}$ be meromorphic functions such that $\phi_{1} \circ p_{1}$ and $\left(\phi_{i} \circ p_{i}\right)^{\prime}, i=1 . . N$ take all complex values but at most a finite number (the hypothesis on $\phi_{i} \circ p_{i}$ could be weakened; even dropped, if $S_{i}$ is parabolic: see [HAY]).

Moreover, let $a_{i}, b_{i}, c_{i}, i=1 . . N$ be complex numbers such that, for each $i, a_{i} \neq 0$ or $b_{i} \neq 0$ or $c_{i} \neq 0$.

Set $S=\prod_{i=1}^{N} S_{i}, \mathcal{U}=\prod_{i=1}^{N}=\mathcal{U}_{i}$ and $p=\left(p_{1} \ldots p_{N}\right)$; consider the meromorphic metric

$$
\Lambda=d \phi_{1} \odot d \phi_{1}+\sum_{i=1}^{N} \frac{d \phi_{i} \odot d \phi_{i}}{a_{i} \phi_{1}^{2}+b_{i} \phi_{1}+c_{i}} .
$$

Theorem 11. $(\mathcal{U}, \Lambda)$ is coercive (hence geodesically complete).

Proof. By pulling back $\Lambda$ with respect to the universal covering $p$ we get

$$
p^{*} \Lambda\left(z^{1} \ldots z^{N}\right)=\left[\left(\phi_{1} \circ p_{1}\right)^{\prime}\right]^{2} d z^{1} \odot d z^{1}+\sum_{i=1}^{N} \frac{\left[\left(\phi_{i} \circ p_{i}\right)^{\prime}\right]^{2} d z^{i} \odot d z^{i}}{a_{i}\left(\phi_{1} \circ p_{1}\right)^{2}+b_{i} \phi_{1} \circ p_{1}+c_{i}} .
$$

We claim that $\left(\mathcal{U}, p^{*} \Lambda\right)$ is coercive: in fact, for every n-tuple $\left(A_{1} \ldots A_{N}\right) \in \mathbb{C}^{N}$ such that

$$
\left\{\begin{array}{l}
\left(\phi_{1} \circ p_{1}\right)^{\prime}(0) \neq 0 \\
A_{1}-\sum_{l=2}^{N} A_{l} a_{i}\left(\phi_{1} \circ p_{1}\right)^{2}+b_{i} \phi_{1} \circ p_{1}+\left.c_{i}\right|_{0} \neq 0
\end{array}\right.
$$

set $\Psi(x):=A_{1}-\sum_{l=2}^{N} A_{l} a_{i}(x)^{2}+b_{i} x+c_{i}$, there holds

$$
\int_{0}^{u^{1}}\left(\Psi\left(\phi_{1} \circ p_{1}(\eta)\right)\right)^{-1 / 2}\left(\phi_{1} \circ p_{1}\right)^{\prime}(\eta) d \eta=\int_{\phi_{1} \circ p_{1}(0)}^{\phi_{1} \circ p_{1}\left(u^{1}\right)}=\Phi\left(\phi_{1} \circ p_{1}\right),
$$

where $\Phi$ is one (depending on the constants $A_{1} \ldots A_{N}$ ) of the holomorphic function germs on the right hand member of proposition 33 .

This fact shows that the maximal analytical continuation of $u^{1} \rightarrow \Phi\left(\phi_{1} \circ p_{1}\left(u^{1}\right)\right)$ takes all $\mathbb{P}^{1}$ 's values but a finite number, because so does the meromorphic function $\phi_{1}$ and hence $\phi_{1} \circ p_{1}$; moreover, for each $i, 2 \leq i \leq N$, each one of the two HFG's $\pm\left[\left(\phi_{i} \circ p_{i}\right)^{\prime}\right]$ could be continuated to $\pm\left[\left(\phi_{i} \circ p_{i}\right)^{\prime}\right]$ which, by assumption, takes all values but at most two ones.

The preceding examples may be readily extended to the following two (alternative) cases, mostly following the outline of the above reasoning:

- $\mathbb{D}^{N}$ taking place of $\mathbb{C}^{N}$ and $h, f_{2} \ldots f_{N}$ meromorphic functions on $\mathbb{D}$ satisfying theorem 9

- $P_{2} \ldots P_{N}$ polynomials of degree at most four: similar conclusions may be drawn by means of elliptic integrals. 


\subsection{Pseudo-Riemannian warped products}

Definition 35. A pseudo-Riemannian manifold is geodesically complete provided that it admits a complexification $M$ such that the Riemann surface, with logarithmic singularities, of each (complexified) geodesic germ is real-complete.

Definition 36. A warped product

$$
\mathcal{U}=\mathcal{U}_{1} \times_{a_{2}\left(u^{1}\right)} \mathcal{U}_{2} \times_{a_{3}\left(u^{1}\right)} \mathcal{U}_{3} \times \ldots \ldots \times_{a_{N}\left(u^{1}\right)} \mathcal{U}_{N}
$$

of real intervals, real lines or $\mathbb{S}^{1}$ 's with nondegenerating real-analytic pseudo-Riemannian metric

$$
\Lambda\left(u^{1} \ldots . . u^{N}\right)=b_{1}\left(u^{1}\right) d u^{i} \odot d u^{i}+\sum_{i=2}^{N} a_{i}\left(u^{i}\right) f_{i}\left(u^{i}\right) d u^{i} \odot d u^{i},
$$

of arbitary signature is coercive provided that, called $\mathcal{K}$ the canonical complexification $\mathbb{R}^{N} \rightarrow \mathbb{C}^{N}$, for one (hence every) point $X_{0}=\left(x_{0}^{1} \ldots x_{0}^{N}\right)$ there holds:

- for every n-tuple $\left(A_{1} \ldots A_{N}\right) \in \mathbb{R}^{N}$ such that $b_{1}\left(x_{0}^{1}\right) \neq 0$ and $A_{1}-\sum_{l=2}^{N} \frac{A_{l}}{a_{l}\left(x_{0}^{1}\right)} \neq 0$ and for each one of the two HFG's $\aleph_{1}$ and $\aleph_{2}$ such that

$$
\left(\boldsymbol{\aleph}_{i}\right)^{2}=\mathcal{K} \circ\left[\frac{1}{b_{1}}\left(A_{1}-\sum_{l=2}^{N} \frac{A_{l}}{a_{l}}\right)\right]_{x_{0}^{1}} \quad i=1,2,
$$

the Riemann surface $\left(S_{1}, \pi_{1}, j_{1}, \Phi_{1}\right)$ of both the HFG's (see remark 21)

$$
\left[\int_{x_{0}}^{u^{1}} \frac{d \eta}{\aleph_{i}(\eta)}\right]_{x_{0}^{1}} \quad i=1,2
$$

is such that $\mathbb{R} \backslash \Phi_{1}\left(S_{1}\right)$ is a finite set;

- for each $k, 2 \leq k \leq N$ and for each one of the two HFG's $\phi_{k 1}$ and $\phi_{k 2}$ such that

$$
\left(\phi_{k i}\right)^{2}=\mathcal{K} \circ\left[f_{k}\right]_{x_{0}^{1}}, \quad i=1,2
$$

the Riemann surface $\left(S_{k}, \pi_{k}, j_{k}, \Phi_{k}\right)$ of both the holomorphic function germs (see remark 21)

$$
\left[\int_{x_{0}^{1}}^{u^{k}} \phi_{k i}(\eta) d \eta\right]_{x_{0}^{1}} i=1,2
$$

is such that $\mathbb{R} \backslash \Phi_{k}\left(S_{k}\right)$ is a finite set.

We confine ourselves in stating the real analogue of our main theorem (the proof is almost identical): 
Theorem 12. A warped product

$$
\mathcal{U}=\mathcal{U}_{1} \times_{a_{2}\left(u^{1}\right)} \mathcal{U}_{2} \times_{a_{3}\left(u^{1}\right)} \mathcal{U}_{3} \times \ldots \ldots \times_{a_{N}\left(u^{1}\right)} \mathcal{U}_{N}
$$

of real intervals, real lines or $\mathbb{S}^{1}$ 's with nondegenerating real-analytic pseudo-Riemannian metric

$$
\Lambda\left(u^{1} \ldots . . u^{N}\right)=b_{1}\left(u^{1}\right) d u^{i} \odot d u^{i}+\sum_{i=2}^{N} a_{i}\left(u^{i}\right) f_{i}\left(u^{i}\right) d u^{i} \odot d u^{i},
$$

of arbitary signature is geodesically complete if and only if it is coercive.

\subsection{The Clifton-Pohl torus}

Consider now $\mathbb{N}:=\mathbb{R}^{2} \backslash\{0\}$, with the Lorentz metric $d u \odot d v /\left(u^{2}+v^{2}\right)$; the group $D$ generated by scalar multiplication by 2 is a group of isometries of $\mathrm{N}$; its action is properly dicontinuous, hence $\mathrm{T}=\mathrm{N} / D$ is a Lorentz surface. Topologically, $\mathrm{T}$ is the closed annulus $1 \leq \varrho \leq 2$, with boundaries identified by the action of $D$, i.e. a torus; notwithstanding, $\mathrm{T}$ is geodesically incomplete, since $t \mapsto(1 /(1-t), 0)$ is a geodesic of $\mathrm{M}$ (see [ONE]). In the following, we shall study directly $\mathrm{N}$ rather than $\mathrm{T}$, since our conclusions could be easily pushed down with respect to the action of $D$. Consider now the holomorphic Riemannian manifold $\mathrm{M}=\left[\mathbb{C}^{2} \backslash((1, i) \mathbb{C} \cup(1,-i) \mathbb{C}), d u \odot d v /\left(u^{2}+v^{2}\right)\right]$.

Lemma 13. The geodesic equations of both $\mathrm{M}$ and $\mathrm{N}$ are: $\ddot{u}=2 u /\left(u^{2}+v^{2}\right) \ddot{u}^{2}, \ddot{v}=$ $2 v /\left(u^{2}+v^{2}\right) \dot{v}^{2}$; they are meant to be real or complex depending on the fact that they concern $\mathrm{M}$ or $\mathrm{N}$.

Proposition 37. All null geodesics of N are complete.

Proof. We may deal with the only case $v=$ const $:=A$. Lemma 13 imply $\ddot{u}=$ $2 u /\left(u^{2}+A^{2}\right) \dot{u}^{2}$, which is solved by $t \mapsto(C-B t)^{-1}$ if $A=0$ and by $t \mapsto \tan (A t+B)$ if $A \neq 0$, for suitable real constants $B$ and $C$. The above functions are restrictions of meromorphic functions, hence, by definition 7 , yield complete geodesics.

We turn to nonnull geodesics of $\mathrm{N}$ :

Lemma 14. The Cauchy's problem $\dot{\varphi}=2 A \operatorname{Ch} \varphi \sqrt{B^{2}-2 / A \operatorname{Ch} \varphi} \varphi(0)=\varphi_{0}$, (with $B^{2}-$ $\left.2 / A \mathrm{Ch} \varphi_{0}>0\right)$ has complete solutions, in the real domain, with respect to the canonical complexification, if and only if $0<A B^{2} \leq 2$.

Proof. Set $F(\varphi)=2 A \operatorname{Ch} \varphi \sqrt{B^{2}-2 / A \operatorname{Ch} \varphi}$ and $G(\varphi):=\int_{\varphi_{0}}^{\varphi} d \nu / F(\nu)$, where by the integral sign we mean the choice of the only primitive of $1 / F$ vanishing at $\varphi_{0}$. Rewrite the problem in the form $G(\varphi)=\boldsymbol{i d}$ : this shows that $\varphi$ and $G$ are inverse elements of holomorphic functions in neighbourhoods of $\varphi_{0}$ and $G\left(\varphi_{0}\right)$.

Suppose $A B^{2} \geq 2$ or $A B^{2}<0$ : then $F$ never vanishes; since $1 / F(\nu)=O\left(e^{-|\nu|}\right)$ as $\nu \rightarrow \infty, G$ takes a bounded set of values, hence, by lemma $2, \varphi$ is not complete.

If, instead, $0<A B^{2} \leq 2$, then there exists a branch of $F$ admitting a zero on the real line, hence there exists a branch $\tilde{f}$ of $1 / F$ whose absolute value takes all large enough 
values. However $\tilde{f}$ can be analytically continued, by admitting complex trips, up to $\left\{\varphi: \operatorname{Ch} \varphi \geq 2 / A B^{2}\right\}$, in such a way that an even function $f$ is yielded.

Now $\left|\int_{\varphi_{0}}^{\varphi} f(\nu) d \nu\right|$ takes all positive values; but $g:=\int_{\varphi_{0}}^{\varphi} f$ is an odd function plus a real constant on $\left\{\varphi: \operatorname{Ch} \varphi \geq 2 / A B^{2}\right\}$, hence it takes all real values with at most the exception of its asympotical value $\sigma$. Thus, if $(S, \varrho, \ell, H)$ is the Riemann surface of $\varphi$, then, by lemma $2, \varrho\left(H^{-1}(\mathbb{R})\right) \cap \mathbb{R} \supset g(\mathbb{R}) \supset \mathbb{R} \backslash\{\sigma\}$.

Definition 38. The impulse function $\boldsymbol{P}: T \mathrm{~N} \backslash\{$ null vectors $\} \rightarrow \mathbb{R}$ is defined by setting $\boldsymbol{P}(\alpha, \beta, x, y)=\left(\alpha^{2}+\beta^{2}\right)^{-1}\left(2 \alpha \beta+\alpha^{2} y / x+\beta x / y\right)$.

Theorem 13. A nonnull geodesic $\gamma$ starting from $(\alpha, \beta)$, with velocity $(x, y)$ is complete if and only if $0<\boldsymbol{P}(\alpha, \beta, x, y) \leq 2$.

Proof. We may suppose $\alpha \neq 0$ and $\beta \neq 0$. Moreover, we have $x \neq 0$ and $y \neq 0$. The equations in lemma 13 can be integrated once to yield:

$$
\dot{u} \dot{v}=A\left(u^{2}+v^{2}\right), \quad u / \dot{u}+v / \dot{v}=B,
$$

where $A=x y /\left(\alpha^{2}+\beta^{2}\right)$ and $B=\alpha / x+\beta / y$; note that $A B^{2}=\boldsymbol{P}(\alpha, \beta, x, y)$.

Introduce now the supplementary hypothesis that $u>0$ and $v>0$ : by performing the change of coordinates $u=e^{\omega}, v=e^{\eta},(5.14)$ is turned into

$$
\dot{\omega} \dot{\eta}=2 A \operatorname{Ch}(\omega-\eta), \quad 1 / \dot{\omega}+1 / \dot{\eta}=B
$$

We can solve with respect to $\dot{\omega}$ and $\dot{\eta}$, getting

$$
\left\{\begin{array}{l}
\dot{\omega}=2\left(B-\sqrt{B^{2}-2 /[A \operatorname{Ch}(\omega-\eta)]}\right)^{-1} \\
\dot{\eta}=2\left(B+\sqrt{B^{2}-2 /[A \operatorname{Ch}(\omega-\eta)]}\right)^{-1} .
\end{array}\right.
$$

Subtract and set $\varphi:=\omega-\eta$; this yields the equation in $\varphi$ studied in lemma 14, with the appropriate initial value $\varphi(0)=\log (u / v)$; this Cauchy's problem has complete solutions if and only if $0<\boldsymbol{P}(\alpha, \beta, x, y) \leq 2$.

Now the fact that $\varphi$ is incomplete easily implies that so is $\gamma$. Suppose, instead, that $\varphi$ is complete: from (5.16), we get that bothm $\dot{\eta}$ and $\dot{\omega}$ is complete; since passing to a primitive preserves completeness, so are $\eta$ and $\omega$ : but $u=e^{\omega}$ and $v=e^{\eta}$ : this eventually implies that $\gamma$ is complete.

To remove the hypothesis that $u>0$ and $v>0$, consider two geodesics $\gamma, \delta$, starting from, say, $(\alpha, 0)$, the former with velocity $(x, y)$ and the latter $(x,-y)(y>0)$. The first order systems, like (5.14), of $\gamma$ and $\delta$ differ only in the signs of constants in their first equations. Thus, the equations of those pieces of $\gamma$ lying in $Q_{1}=\{u>0, v>0\}$ and of those ones of $\delta$ lying in $Q_{2}=\{u>0, v<0\}$ are transformed into the same system (5.15) by performing the change of coordinates $(u, v)=\left(e^{\omega}, e^{\eta}\right)$ in $Q_{1}$, resp. $(u, v)=\left(e^{\omega},-e^{\eta}\right)$ in $Q_{2}$; an analogous argument holds for the other octants. It is easily seen that if a nonnull geodesic intersects one of the coordinate axes at a point $P$, it does with finite (nonnull) velocity, hence it can be analytically continued across $P$, changing octant: thus, once obtained the (maximal) curve $t \mapsto(\omega(t), \eta(t))$, we can reconstruct the original (maximal) geodesic $t \mapsto(u(t), v(t))$ by choosing the only smooth curve starting from $(\alpha, \beta)$ whose graph is contained in the set $\left(t, u, v \in \mathbb{R}^{3}\right): u= \pm \boldsymbol{e}^{\omega(t)}, v= \pm \boldsymbol{e}^{\eta(t)}$. 


\section{References}

[BEH] John K.Beem, Paul E.Ehrlich, Global Lorentzian geometry Marcel Dekker, 1981

[BFV] Andrzej Borowiec, Mauro Francaviglia, Igor Volovichy Anti Kählerian Manifolds, Differential Geometry and Its Applications 12 (2000), 281-289.

[CAS] Antonio Cassa, Teoria delle curve algebriche piane e delle superfici di Riemann compatte, Pitagora, 1983.

[CSB] Antonio Cassa, Reconstructing space-time from world-lines of charged particles, Journal of Geometry and Physics 19 (1996) 77-89.

[CSC] Antonio Cassa, A bridge between complex geometry and Riemannian geometry Proc. of the Amer. Math. Soc. 2 (1993), 621-627.

[CR] Carrière, Yves, Rozoy, Luc, Complétude des métrique lorentziennes de $T^{2}$ et difféomorphismes du cercle Bol.Soc.Brasil.Matt. N.S. 25 (1994).

[DNF] B A Dubrovin, S P Novikov, A T Fomenko, Geometria delle superfici dei gruppi di trasformazioni e dei campi, Editori riuniti, 1987.

[FO] Otto Forster, Lectures on Riemann surfaces, Springer Verlag, 1981.

[GAR] P R Garabedian, Partial differential equations, John Wiley \& sons, 1964.

[GRO] Robert C Gunning, Hugo Rossi, Analytic functions of several complex variables, Prentice Hall, 1965.

[HAY] W K Hayman, Meromorphic functions, Oxford at the Clarendon press, 1964.

[HIL] Einar Hille, Ordinary differential equations in the complex domain, John Wiley \& sons, 1976.

[INC] E L Ince, Ordinary differential equations, Dover, 1956 (originally published in 1926).

[LEB] Claude Lebrun, Spaces of complex null geodesics in complex-Riemannian geometry, Trans. of the AMS, vol $278 \mathrm{nr}$ 1, July 1983.

[MAN] Yuri Manin, Gauge fields theory and complex geometry, Springer Verlag, 1984.

[NEV] Rolf Nevanlinna, Le théorème de Picard-Borel et la théorie des fonctions méromorphes, Chelsea publishing company, Bronx, New York (originally published Paris, 1939).

[ONE] Barret O'Neill, Semi-Riemannian geometry, Academic Press, 1983

[RS] Alfonso Romero, Miguel Sanchez On the completeness of geodesic obtained as a limit, J Math Phys 34 (1993), 8.

[SPR] George Springer, Introduction to Riemann surfaces, Addison-Wesley, 1957.

[VRA] G Vranceanu, Leçons de géométrie différentielle,, Editions de l'académie de la république populaire roumaine, 1957.

[WEL] R O Wells, Differential analysis on complex manifolds, Prentice-Hall, 1973 